MATHEMATICS OF COMPUTATION

Volume 72, Number 242, Pages 865-889

S 0025-5718(02)01470-9

Article electronically published on October 18, 2002

\title{
RELATIVE INVARIANTS \\ OF SOME 2-SIMPLE PREHOMOGENEOUS VECTOR SPACES
}

\author{
TAKEYOSHI KOGISO, GO MIYABE, MIYUKI KOBAYASHI, AND TATSUO KIMURA
}

\begin{abstract}
In this paper, we shall construct explicitly irreducible relative invariants of two 2 -simple prehomogeneous vector spaces. Together with a preprint by the same authors, this completes the list of all relative invariants of regular 2-simple prehomogeneous vector spaces of type I.
\end{abstract}

\section{INTRODUCTION}

Let $G$ be a connected reductive algebraic group defined over the complex number field $\mathbb{C}, V$ a finite dimensional vector space, and $\rho: G \longrightarrow G L(V)$ a rational representation of $G$. Such a triplet $(G, \rho, V)$ is called a prehomogeneous vector space (abbreviated P.V.) if $V$ has an open $G$-orbit, and a triplet is called irreducible if $\rho$ is an irreducible representation. Furthermore, such a triplet $(G, \rho, V)$ is called simple (resp. $n$-simple) if the derived subgroup $[G, G]$ is a simple algebraic group (resp. the product of $n$-simple algebraic groups). A nonzero rational function $F(x)$ is called a relative invariant corresponding to a character $\chi: G \rightarrow G L_{1}$ if it satisfies the relation $F(\rho(g) x)=\chi(g) F(x)$ as a rational function for all $g \in G$.

A complete list of irreducible prehomogeneous vector spaces is given by M. Sato and T. Kimura in [1]. At the same time, the relative invariants are constructed for almost all of these spaces. However, for some complicated prehomogeneous vector spaces, such as classification numbers (6), (7), (10), (20), (21) and (24) in [1], the construction of relative invariants had not been settled. In 1971, an irreducible relative invariant of (20) was constructed in [6] and, in 1981, that of (6), (7) was constructed in [3]. In 1990, relative invariants for (10), (21), (24) were constructed in [7] by some complicated calculations. In 1995, that of (10) and (21) was constructed in [8] by using the notion of the quotient space. For the case of nonirreducible prehomogeneous vector spaces, in 1983, T. Kimura studied the case of nonirreducible simple prehomogeneous vector spaces. In 1988, T. Kimura, S. Kasai, M. Inuzuka and O. Yasukura 9] completed the classifications of nonirreducible reduced 2-simple prehomogeneous vector spaces of type I. See 9 for the definition of type I and type II.

Recently, the relative invariants are constructed in [10] and 11] for almost all of these spaces of type I except for the following two cases:

(regular 8) $\left(G L_{1}^{2} \times S L_{5} \times S L_{8}, \Lambda_{2} \otimes \Lambda_{1}+1 \otimes \Lambda_{1}^{*}, \mathrm{Alt}_{5}^{\oplus 8} \oplus V(8)^{*}\right)$,

(regular 40) $\left(G L_{1}^{2} \times \operatorname{Spin}_{10} \times S L_{14},(\right.$ a half-spin rep. $) \otimes \Lambda_{1}+1 \otimes \Lambda_{1}^{*}, V(16)^{\oplus 14} \oplus$ $\left.V(14)^{*}\right)$.

Received by the editor April 20, 2000 and, in revised form, May 29, 2001.

2000 Mathematics Subject Classification. Primary 11S05, 11S90.

(C)2002 American Mathematical Society 
These cases are the most complicated and difficult cases in 9. The purpose of this paper is to construct explicitly irreducible relative invariants of the above 2 cases and to complete the construction of irreducible relative invariants for all 2-simple prehomogeneous vector spaces of type I. In this paper, we have reduced this construction problem to determine some polynomials. Although we used the computer software Mathematica [12] to decide the explicit form of polynomials, once we obtain them, it is not necessary to use the computer to check them.

\section{Notations AND PRELIMINARIES}

We denote by Alt $_{n}\left(\right.$ resp. Sym ${ }_{n}$ ) the totality of $n \times n$ alternating matrices (resp. $n \times n$ symmetric matrices). For $X \in \operatorname{Alt}_{2 n}$, let $P f(X)$ be the Pfaffian of $X$ so that we have $P f(X)^{2}=\operatorname{det} X$ and $P f\left(A X^{t} A\right)=\operatorname{det} A \cdot P f(X)$ for $A \in G L_{2 n}$. We denote $\Lambda^{\prime}$ (resp. $\chi$ ) the even half-spin representation (resp. the vector representation) of $\operatorname{Spin}_{10}$. Note that the dual representation $\Lambda^{\prime *}$ of $\Lambda^{\prime}$ is the odd half-spin representation of $\operatorname{Spin}_{10}$. For the infinitesimal representation of $\Lambda^{\prime}$, see (5.38) in [1].

For $X \in M_{n}$ (=the totality of $n \times n$ matrices), let $\Delta(X)$ be the cofactor matrix of $X$ so that we have $X \cdot \Delta(X)=\Delta(X) \cdot X=\operatorname{det} X \cdot I_{n}$.

When we prove the irreducibility of relative invariants, we often use the following facts.

Lemma 2.1 (cf. [1]). Let $(G, \rho, V)$ be a P.V. with a generic point $v_{0}$.

(1) For a character $\chi: G \rightarrow G L_{1}$, there exists a relative invariant corresponding to $\chi$ if and only if $\left.\chi\right|_{G_{v_{0}}}=1$, where $G_{v_{0}}=\left\{g \in G ; \rho(g) v_{0}=v_{0}\right\}$.

(2) Any irreducible component of a relative invariant is also a relative invariant.

Lemma 2.2 (cf. [1], $\ 4$ Proposition 18). There is a one-to-one correspondence between relative invariants $f(x)$ of $\left(G \times S L_{n}, \rho \otimes \Lambda_{1}, V(m) \otimes V(n)\right)(m>n \geq 1)$ and relative invariants $\tilde{f}(\tilde{x})$ of its castling transform $\left(G \times S L_{m-n}, \rho^{*} \otimes \Lambda_{1}, V(m)^{*} \otimes\right.$ $V(m-n))$. Moreover, there exists a positive integer $d$ for each $f(x)$ such that $\operatorname{deg} f(x)=n d$ and $\operatorname{deg} \tilde{f}(\tilde{x})=(m-n) d$. If $f$ is irreducible, then $\tilde{f}$ is also irreducible.

Moreover, we prove the following lemma to construct some $G$-equivariant mapping in the first example.

Lemma 2.3. For $X=\left(x_{i j}\right)=\left(x_{1}|\cdots| x_{n}\right) \in M(n+2, n)$, let $X^{(i, j)} \in M(n)$ be the matrix obtained from $X$ by subtracting $i$-th and $j$-th rows. For $i<j$, put $\tilde{x_{i j}}=$ $(-1)^{i+j+1} \operatorname{det} X^{(i, j)}$ and define the alternating matrix $\varphi(X)=\left(\tilde{x_{i j}}\right) \in \mathrm{Alt}_{n+2}$. Then the map $\varphi: M(n+2, n) \rightarrow$ Alt $_{n+2}$ satisfies $\varphi(A X)=\operatorname{det} A \cdot{ }^{t} A^{-1} \varphi(X) A^{-1}$ for $A \in G L_{n+2}$.

Proof. Put $Y=A X=\left(y_{1}|\cdots| y_{n}\right)$. For $i<j$, we have

$$
\begin{aligned}
& e_{i} \wedge e_{j} \wedge x_{1} \wedge \cdots \wedge x_{n} \\
& \quad=\operatorname{det} X^{(i, j)} e_{i} \wedge e_{j} \wedge e_{1} \wedge \cdots \wedge e_{i-1} \wedge e_{i+1} \wedge \cdots \wedge e_{j-1} \wedge e_{j+1} \wedge \cdots \wedge e_{n+2} \\
& \quad=\tilde{x_{i j}} e_{1} \wedge \cdots \wedge e_{n+2} .
\end{aligned}
$$


Then, by the action of $A=\left(a_{i j}\right) \in G L_{n+2}$, we have

$$
\begin{aligned}
\tilde{x}_{i j} \operatorname{det} & A \cdot e_{1} \wedge \cdots \wedge e_{n+2}=\tilde{x_{i j}}\left(A e_{1}\right) \wedge \cdots \wedge\left(A e_{n+2}\right) \\
= & \left(\sum_{l=1}^{n+2} e_{l} a_{l i}\right) \wedge\left(\sum_{k=1}^{n+2} e_{k} a_{k i}\right) \wedge y_{1} \wedge \cdots \wedge y_{n} \\
= & \sum_{l<k} a_{l i} a_{k j} e_{l} \wedge e_{k} \wedge y_{1} \wedge \cdots \wedge y_{n}-\sum_{k<l} a_{l i} a_{k j} e_{k} \wedge e_{l} \wedge y_{1} \wedge \cdots \wedge y_{n} \\
= & \sum_{l<k}\left(a_{l i} a_{k j}-a_{k i} a_{l j}\right) e_{l} \wedge e_{k} \wedge y_{1} \wedge \cdots \wedge y_{n} \\
= & \sum_{l<k}\left(a_{l i} a_{k j}-a_{k i} a_{l j}\right) \tilde{y_{l k}} \wedge e_{1} \wedge \cdots \wedge e_{n+2} .
\end{aligned}
$$

Hence, we have $\sum_{l<k}\left(a_{l i} a_{k j}-a_{k i} a_{l j}\right) \tilde{y_{l k}}=\operatorname{det} A \cdot \tilde{x_{i j}}$, which is equivalent to ${ }^{t} A \varphi(Y) A=\operatorname{det} A \cdot \varphi(X)$. This implies that $\varphi(A X)=\operatorname{det} A \cdot{ }^{t} A^{-1} \varphi(X) A^{-1}$.

\section{EXPLICIT CONSTRUCTION OF IRREDUCIBLE RELATIVE INVARIANTS}

In this section, $H$ denotes the generic isotropy subgroup of a P.V. $(G, \rho, V)$, and $H_{1} \sim H_{2}$ implies that $H_{1}$ and $H_{2}$ are locally isomorphic, namely their Lie algebras are isomorphic. $N$ denotes the number of the basic irreducible relative invariants.

3.1. Explicit construction of irreducible relative invariants of $\left(G L_{1}^{2} \times S L_{5} \times S L_{8}, \Lambda_{2} \otimes \Lambda_{1}+1 \otimes \Lambda_{1}^{*}\right)$ with $H \sim S O_{2}, N=2$. To investigate relative invariants, we may assume that $G=G L_{5} \times G L_{8}$ acts on $V=\operatorname{Alt}_{5}^{\oplus 8} \oplus M(8,1)$ by

$$
x=\left(\left(X_{1}, X_{2}, \cdots, X_{8}\right), Y\right) \mapsto\left(\left(A X_{1}{ }^{t} A, \cdots, A X_{8}{ }^{t} A\right)^{t} B,{ }^{t} B^{-1} Y\right)
$$

for $x=\left(\left(X_{1}, \cdots, X_{8}\right), Y\right) \in V$ and $g=(A, B) \in G$. We shall construct the irreducible relative invariants of this prehomogeneous vector space by the following steps.

Step 1. For $Y={ }^{t}\left(y_{1}, \cdots, y_{8}\right) \in M(8,1)$, we put $X \cdot Y=\sum_{i=1}^{8} y_{i} X_{i} \in \mathrm{Alt}_{5}$. Then we obtain that

$$
X \cdot Y \mapsto A X \cdot Y^{t} A
$$

and hence we have

$$
\Delta(X \cdot Y) \mapsto(\operatorname{det} A)^{2 t} A^{-1} \Delta(X \cdot Y) A^{-1},
$$

where $\Delta(X \cdot Y)$ is the cofactor matrix of the odd size alternating matrix the $X \cdot Y \in$ Alt $_{5}$. Note that $\Delta(X \cdot Y) \in \mathrm{Sym}_{5}$.

Step 2. For

$$
X_{i}=\left(\begin{array}{ccccc}
0 & x_{12}^{(i)} & x_{13}^{(i)} & x_{14}^{(i)} & x_{15}^{(i)} \\
-x_{12}^{(i)} & 0 & x_{23}^{(i)} & x_{24}^{(i)} & x_{25}^{(i)} \\
-x_{13}^{(i)} & -x_{23}^{(i)} & 0 & x_{34}^{(i)} & x_{35}^{(i)} \\
-x_{14}^{(i)} & -x_{24}^{(i)} & -x_{34}^{(i)} & 0 & x_{45}^{(i)} \\
-x_{15}^{(i)} & -x_{25}^{(i)} & -x_{35}^{(i)} & -x_{45}^{(i)} & 0
\end{array}\right) \in \operatorname{Alt}_{5}(i=1,2, \cdots, 8)
$$

we put $\tilde{X}_{i}:={ }^{t}\left(x_{12}^{(i)}, x_{13}^{(i)}, x_{14}^{(i)}, x_{15}^{(i)}, x_{23}^{(i)}, x_{24}^{(i)}, x_{25}^{(i)}, x_{34}^{(i)}, x_{35}^{(i)}, x_{45}^{(i)}\right) \in M(10,1)$. 
The action $X_{i} \mapsto A X_{i}{ }^{t} A$ induces $\tilde{X}_{i} \mapsto \Lambda_{2}(A) \tilde{X}_{i} \quad\left(\Lambda_{2}(A) \in G L_{10}\right)$. Then we put $Z=\left(\tilde{X}_{1}, \tilde{X}_{2}, \ldots, \tilde{X}_{8}\right) \in M(10,8)$ and define the $10 \times 10$-alternating matrix $\tilde{Z}=$ $\left(z_{i j}\right)_{1 \leq i<j \leq 10}$ with $z_{i j}=(-1)^{i+j} \operatorname{det} Z^{(i, j)}$, where $Z^{(i, j)}$ is the $8 \times 8$-matrix obtained from $Z$ by substracting the $i$-th and the $j$-th rows. Then, by Lemma 2.3 , we have $\tilde{Z} \mapsto\left(\operatorname{det} \Lambda_{2}(A)\right)(\operatorname{det} B)^{t} \Lambda_{2}(A)^{-1} \tilde{Z} \Lambda_{2}(A)^{-1}$. Note that $\operatorname{det} \Lambda_{2}(A)=(\operatorname{det} A)^{4}$.

Step 3. If we put $\Phi(\tilde{Z}):=\left(\varphi(\tilde{Z})_{i, j}\right)_{1 \leq i \leq j \leq 5} \in \operatorname{Sym}_{5}$ for $\tilde{Z} \in$ Alt $_{10}$ with the following entries, then we have Lemma 3.1.

$$
\begin{aligned}
& \varphi_{11}=z_{510}^{2}+z_{69}^{2}+z_{78}^{2}-2 z_{59} z_{610}-2 z_{68} z_{79}+2 z_{58} z_{710}-2 z_{67} z_{89}+2 z_{57} z_{810}-2 z_{56} z_{910}, \\
& \varphi_{22}=z_{210}^{2}+z_{39}^{2}+z_{48}^{2}-2 z_{29} z_{310}+2 z_{28} z_{410}-2 z_{38} z_{49}+2 z_{24} z_{810}-2 z_{34} z_{89}-2 z_{23} z_{910}, \\
& \varphi_{33}=z_{110}^{2}+z_{37}^{2}+z_{46}^{2}-2 z_{17} z_{310}-2 z_{36} z_{47}+2 z_{16} z_{410}-2 z_{34} z_{67}+2 z_{14} z_{610}-2 z_{13} z_{710} \text {, } \\
& \varphi_{44}=z_{19}^{2}+z_{27}^{2}+z_{45}^{2}-2 z_{17} z_{29}-2 z_{25} z_{47}+2 z_{15} z_{49}-2 z_{24} z_{57}+2 z_{14} z_{59}-2 z_{12} z_{79} \text {, } \\
& \varphi_{55}=z_{18}^{2}+z_{26}^{2}+z_{35}^{2}-2 z_{16} z_{28}-2 z_{25} z_{36}+2 z_{15} z_{38}-2 z_{23} z_{56}+2 z_{13} z_{58}-2 z_{12} z_{68} \text {, } \\
& \varphi_{12}=-z_{210} z_{510}-z_{410} z_{58}+z_{310} z_{59}+z_{29} z_{610}+z_{49} z_{68}-z_{39} z_{69} \\
& -z_{28} z_{710}-z_{48} z_{78}+z_{38} z_{79}-z_{27} z_{810}+z_{45} z_{810}+z_{37} z_{89}-z_{46} z_{89}+z_{26} z_{910}-z_{35} z_{910} \text {, } \\
& \varphi_{13}=z_{110} z_{510}+z_{410} z_{56}-z_{310} z_{57}-z_{19} z_{610}-z_{45} z_{610}+z_{39} z_{67} \\
& -z_{48} z_{67}-z_{47} z_{68}+z_{46} z_{69}+z_{18} z_{710}+z_{35} z_{710}+z_{37} z_{78}-z_{36} z_{79}+z_{17} z_{810}-z_{16} z_{910}, \\
& \varphi_{14}=z_{45} z_{510}-z_{49} z_{56}+z_{210} z_{57}+z_{48} z_{57}+z_{47} z_{58}-z_{110} z_{59} \\
& -z_{46} z_{59}-z_{29} z_{67}+z_{19} z_{69}-z_{25} z_{710}-z_{27} z_{78}-z_{18} z_{79}+z_{26} z_{79}-z_{17} z_{89}+z_{15} z_{910}, \\
& \varphi_{15}=-z_{35} z_{510}-z_{210} z_{56}+z_{39} z_{56}-z_{38} z_{57}+z_{110} z_{58}-z_{37} z_{58} \\
& +z_{36} z_{59}+z_{25} z_{610}+z_{28} z_{67}-z_{19} z_{68}+z_{27} z_{68}-z_{26} z_{69}+z_{18} z_{78}-z_{15} z_{810}+z_{16} z_{89}, \\
& \varphi_{23}=-z_{110} z_{210}+z_{19} z_{310}+z_{27} z_{310}-z_{37} z_{39}-z_{18} z_{410}-z_{26} z_{410} \\
& +z_{38} z_{47}-z_{46} z_{48}+z_{36} z_{49}-z_{24} z_{610}+z_{34} z_{69}+z_{23} z_{710}-z_{34} z_{78}-z_{14} z_{810}+z_{13} z_{910} \text {, } \\
& \varphi_{24}=-z_{210} z_{27}+z_{110} z_{29}+z_{29} z_{37}-z_{19} z_{39}+z_{25} z_{410}-z_{28} z_{47} \\
& +z_{45} z_{48}+z_{18} z_{49}-z_{35} z_{49}+z_{24} z_{510}-z_{34} z_{59}+z_{24} z_{78}-z_{23} z_{79}+z_{14} z_{89}-z_{12} z_{910}, \\
& \varphi_{25}=z_{210} z_{26}-z_{110} z_{28}-z_{25} z_{310}-z_{29} z_{36}+z_{19} z_{38}+z_{35} z_{39} \\
& -z_{38} z_{45}+z_{28} z_{46}-z_{18} z_{48}-z_{23} z_{510}+z_{34} z_{58}-z_{24} z_{68}+z_{23} z_{69}+z_{12} z_{810}-z_{13} z_{89} \text {, } \\
& \varphi_{34}=-z_{110} z_{19}+z_{17} z_{210}-z_{27} z_{37}+z_{17} z_{39}-z_{15} z_{410}-z_{45} z_{46} \\
& +z_{26} z_{47}+z_{35} z_{47}-z_{16} z_{49}-z_{14} z_{510}+z_{34} z_{57}+z_{24} z_{67}-z_{14} z_{69}+z_{12} z_{710}+z_{13} z_{79}, \\
& \varphi_{35}=z_{110} z_{18}-z_{16} z_{210}+z_{15} z_{310}+z_{27} z_{36}-z_{35} z_{37}-z_{17} z_{38} \\
& +z_{36} z_{45}-z_{26} z_{46}+z_{16} z_{48}+z_{13} z_{510}-z_{34} z_{56}-z_{12} z_{610}-z_{23} z_{67}+z_{14} z_{68}-z_{13} z_{78}, \\
& \varphi_{45}=-z_{18} z_{19}-z_{26} z_{27}+z_{17} z_{28}+z_{16} z_{29}+z_{25} z_{37}-z_{15} z_{39} \\
& -z_{35} z_{45}+z_{25} z_{46}-z_{15} z_{48}+z_{24} z_{56}+z_{23} z_{57}-z_{14} z_{58}-z_{13} z_{59}+z_{12} z_{69}+z_{12} z_{78} \text {. }
\end{aligned}
$$

Lemma 3.1. For every $A \in G L_{5}, B \in G L_{8}, \quad \tilde{Z} \in \mathrm{Alt}_{10}$, we have

$$
\tilde{Z} \mapsto(\operatorname{det} A)^{4}(\operatorname{det} B)^{t} \Lambda_{2}(A)^{-1} \tilde{Z} \Lambda_{2}(A)^{-1}
$$

and

$$
\Phi\left((\operatorname{det} A)^{4}(\operatorname{det} B)^{t} \Lambda_{2}(A)^{-1} \tilde{Z} \Lambda_{2}(A)^{-1}\right)=(\operatorname{det} A)^{6}(\operatorname{det} B)^{2} \cdot A \Phi(\tilde{Z})^{t} A .
$$


Proof. It is enough to prove the equivariance (3.3) in the case when $A$ is one of the fundamental matrices

$$
A_{u}=\left(\begin{array}{ccccc}
1 & \varepsilon & & & \\
& 1 & & & \\
& & 1 & & \\
& & & 1 & \\
& & & & 1
\end{array}\right), A_{d}=\operatorname{diag}(a, 1,1,1,1),
$$

or permutation matrices.

Checking (3.3) for diagonal or permutation matrices is easy. Note that $\operatorname{det} A_{d}=a$ and $\Phi\left(a^{4 t} \Lambda_{2}\left(A_{d}\right)^{-1} \tilde{Z} \Lambda_{2}\left(A_{d}\right)^{-1}\right)=a^{6} A_{d} \Phi(\tilde{Z})^{t} A_{d}$. For $A_{u}$, we consider the action of $A_{u}$. Since $\operatorname{det} A_{u}=1$,

$$
\Phi(\tilde{Z}) \mapsto \Phi\left({ }^{t} \Lambda_{2}\left(A_{u}\right)^{-1} \tilde{Z} \Lambda_{2}\left(A_{u}\right)^{-1}\right) .
$$

Then we have $\varphi_{11} \mapsto \varphi_{11}+2 \varepsilon \varphi_{12}+\varepsilon^{2} \varphi_{22}, \quad \varphi_{1 j} \mapsto \varphi_{1 j}+\varepsilon \varphi_{2 j}(2 \leq j \leq 5), \quad \varphi_{l k} \mapsto$ $\varphi_{l k}(2 \leq l \leq k \leq 5)$. Hence, we have $\Phi\left({ }^{t} \Lambda_{2}\left(A_{u}\right)^{-1} \tilde{Z} \Lambda_{2}\left(A_{u}\right)^{-1}\right)=A_{u} \Phi(\tilde{Z})^{t} A_{u}$.

Remark A. By using the computer software Mathematica [12, we calculate the above polynomials $\varphi_{i j}(1 \leq i \leq j \leq 5)$ along the following program.

(i) First, we construct the polynomial $\varphi_{11}$. For the action of the diagonal matrix $\operatorname{diag}\left(a_{1}, a_{2}, a_{3}, a_{4}, a_{5}\right)$, denote by $T_{1}$ the polynomial corresponding to the weight $a_{1}^{2}(\operatorname{det} A)^{10}$ :

$$
\begin{aligned}
T_{1}= & k_{1} z_{56} z_{910}+k_{2} z_{57} z_{810}+k_{3} z_{58} z_{710}+k_{4} z_{59} z_{610}+k_{5} z_{510}^{2}+k_{6} z_{510} z_{69} \\
& +k_{7} z_{510} z_{78}+k_{8} z_{69}^{2}+k_{9} z_{69} z_{78}+k_{10} z_{78}^{2}+k_{11} z_{67} z_{89}+k_{12} z_{68} z_{79} .
\end{aligned}
$$

(ii) Next, we calculate the invariant polynomial $T T_{1}$ under the action of permutation matrices (23), (24), (25), (35), (45) on $T_{1}$ :

$$
\begin{aligned}
T T_{1}= & l_{1}\left(z_{56} z_{910}+z_{67} z_{89}-z_{57} z_{810}+z_{68} z_{79}-z_{58} z_{710}+z_{59} z_{610}\right) \\
& +l_{2}\left(z_{510}^{2}+z_{69}^{2}+z_{78}^{2}\right) .
\end{aligned}
$$

(iii) Next, we calculate the invariant polynomial $T T T_{1}$ under the action of unipotent matrices:

$$
\begin{gathered}
\left(\begin{array}{lllll}
1 & & & & \\
& 1 & \varepsilon & & \\
& & 1 & & \\
& & & 1 & \\
& & & & 1
\end{array}\right), \\
\left(\begin{array}{cccccc}
1 & & & & \\
& 1 & & & \\
& & 1 & \varepsilon & \\
& & & 1 & \\
& & & & 1
\end{array}\right),
\end{gathered}
$$

and we can construct the polynomial $\varphi_{11}$ uniquely up to the constant

$$
\begin{aligned}
\varphi_{11}= & z_{510}^{2}+z_{69}^{2}+z_{78}^{2}-2 z_{59} z_{610}-2 z_{68} z_{79} \\
& -2 z_{58} z_{710}-2 z_{67} z_{89}-2 z_{57} z_{810}-2 z_{56} z_{910} .
\end{aligned}
$$

(iv) From the explicit form of $\varphi_{11}$, we can construct the other $\varphi_{i j}$ by the action of the generators of $G L_{5}$ on $\varphi_{11}$. 
Step 4. If we put $\Psi(\tilde{Z}):=\left(\psi(\tilde{z})_{i, j}\right)_{1 \leq i<j \leq 5} \in \operatorname{Alt}_{5}$ for $\tilde{Z} \in$ Alt $_{10}$ with the entries below, then we have Lemma 3.2 .

$$
\begin{aligned}
& \psi_{12}=-z_{110} z_{15} z_{210}+z_{110}^{2} z_{25}-z_{110} z_{19} z_{26}+2 z_{17} z_{210} z_{26}+z_{110} z_{18} z_{27} \\
& -2 z_{16} z_{210} z_{27}-z_{110} z_{17} z_{28}+z_{110} z_{16} z_{29}+z_{15} z_{19} z_{310}-3 z_{17} z_{25} z_{310} \\
& +2 z_{15} z_{27} z_{310}-z_{110} z_{19} z_{35}+z_{17} z_{210} z_{35}+z_{19}^{2} z_{36}+2 z_{27}^{2} z_{36} \\
& -3 z_{17} z_{29} z_{36}-z_{18} z_{19} z_{37}-2 z_{26} z_{27} z_{37}+z_{17} z_{28} z_{37}+2 z_{16} z_{29} z_{37} \\
& -2 z_{27} z_{35} z_{37}+2 z_{25} z_{37}^{2}+z_{17} z_{19} z_{38}-z_{17} z_{27} z_{38}-z_{16} z_{19} z_{39}+z_{17} z_{26} z_{39} \\
& +2 z_{17} z_{35} z_{39}-2 z_{15} z_{37} z_{39}-z_{15} z_{18} z_{410}+3 z_{16} z_{25} z_{410} \\
& -2 z_{15} z_{26} z_{410}-z_{15} z_{35} z_{410}+z_{110} z_{18} z_{45}-z_{16} z_{210} z_{45}+z_{15} z_{310} z_{45} \\
& +2 z_{27} z_{36} z_{45}-2 z_{35} z_{37} z_{45}-2 z_{17} z_{38} z_{45}+2 z_{36} z_{45}^{2}-z_{18} z_{19} z_{46}-2 z_{26} z_{27} z_{46} \\
& +2 z_{17} z_{28} z_{46}+z_{16} z_{29} z_{46}+2 z_{25} z_{37} z_{46}-z_{15} z_{39} z_{46}-2 z_{26} z_{45} z_{46}-2 z_{35} z_{45} z_{46} \\
& +2 z_{25} z_{46}^{2}+z_{18}^{2} z_{47}+2 z_{26}^{2} z_{47}-3 z_{16} z_{28} z_{47}+2 z_{26} z_{35} z_{47}+2 z_{35}^{2} z_{47}-6 z_{25} z_{36} z_{47} \\
& +3 z_{15} z_{38} z_{47}-z_{17} z_{18} z_{48}+z_{16} z_{27} z_{48}-z_{15} z_{37} z_{48}+2 z_{16} z_{45} z_{48} \\
& -2 z_{15} z_{46} z_{48}+z_{16} z_{18} z_{49}-z_{16} z_{26} z_{49}-2 z_{16} z_{35} z_{49} \\
& +3 z_{15} z_{36} z_{49}+z_{110} z_{12} z_{510}-z_{17} z_{23} z_{510}+z_{16} z_{24} z_{510}-z_{14} z_{26} z_{510} \\
& +z_{13} z_{27} z_{510}-2 z_{14} z_{35} z_{510}+2 z_{13} z_{45} z_{510}-z_{14} z_{210} z_{56}-z_{27} z_{34} z_{56} \\
& +z_{24} z_{37} z_{56}+z_{14} z_{39} z_{56}+z_{12} z_{410} z_{56}-z_{34} z_{45} z_{56}+z_{24} z_{46} z_{56} \\
& -2 z_{23} z_{47} z_{56}-z_{13} z_{49} z_{56}+z_{13} z_{210} z_{57}-z_{12} z_{310} z_{57}+z_{26} z_{34} z_{57}+z_{34} z_{35} z_{57} \\
& -2 z_{24} z_{36} z_{57}+z_{23} z_{37} z_{57}-z_{14} z_{38} z_{57}+z_{23} z_{46} z_{57}+z_{13} z_{48} z_{57}+z_{110} z_{14} z_{58} \\
& +z_{17} z_{34} z_{58}-2 z_{14} z_{37} z_{58}-z_{14} z_{46} z_{58}+3 z_{13} z_{47} z_{58}-z_{110} z_{13} z_{59}-z_{16} z_{34} z_{59} \\
& +3 z_{14} z_{36} z_{59}-z_{13} z_{37} z_{59}-2 z_{13} z_{46} z_{59}-z_{12} z_{19} z_{610}-z_{15} z_{24} z_{610} \\
& +3 z_{14} z_{25} z_{610}-z_{12} z_{27} z_{610}-2 z_{12} z_{45} z_{610}+z_{24} z_{26} z_{67}-z_{23} z_{27} z_{67}+z_{14} z_{28} z_{67} \\
& -z_{13} z_{29} z_{67}-2 z_{25} z_{34} z_{67}+z_{24} z_{35} z_{67}+z_{12} z_{39} z_{67}-z_{23} z_{45} z_{67}-z_{12} z_{48} z_{67} \\
& -z_{14} z_{19} z_{68}-z_{17} z_{24} z_{68}+2 z_{14} z_{27} z_{68}+z_{14} z_{45} z_{68}-3 z_{12} z_{47} z_{68}+z_{13} z_{19} z_{69} \\
& +z_{17} z_{23} z_{69}-2 z_{14} z_{26} z_{69}+z_{15} z_{34} z_{69}-z_{14} z_{35} z_{69}+z_{12} z_{37} z_{69} \\
& +2 z_{12} z_{46} z_{69}+z_{12} z_{18} z_{710}+z_{15} z_{23} z_{710}-3 z_{13} z_{25} z_{710}+z_{12} z_{26} z_{710} \\
& +2 z_{12} z_{35} z_{710}+z_{14} z_{18} z_{78}+z_{16} z_{24} z_{78}-2 z_{13} z_{27} z_{78}-z_{15} z_{34} z_{78} \\
& +2 z_{12} z_{37} z_{78}-z_{13} z_{45} z_{78}+z_{12} z_{46} z_{78}-z_{13} z_{18} z_{79}-z_{16} z_{23} z_{79} \\
& +2 z_{13} z_{26} z_{79}+z_{13} z_{35} z_{79}-3 z_{12} z_{36} z_{79}-2 z_{14} z_{15} z_{810}+2 z_{12} z_{17} z_{810} \\
& +2 z_{14} z_{16} z_{89}-2 z_{13} z_{17} z_{89}+2 z_{13} z_{15} z_{910}-2 z_{12} z_{16} z_{910} \\
& \psi_{13}=-z_{15} z_{210}^{2}+z_{110} z_{210} z_{25}+z_{19} z_{210} z_{26}-z_{18} z_{210} z_{27}-2 z_{110} z_{19} z_{28}+z_{17} z_{210} z_{28} \\
& +2 z_{110} z_{18} z_{29}-z_{16} z_{210} z_{29}-2 z_{19} z_{25} z_{310}-z_{25} z_{27} z_{310} \\
& +3 z_{15} z_{29} z_{310}+z_{210} z_{27} z_{35}-z_{110} z_{29} z_{35}-z_{19} z_{29} z_{36}+z_{27} z_{29} z_{36}-z_{27} z_{28} z_{37} \\
& +z_{18} z_{29} z_{37}-2 z_{29} z_{35} z_{37}+2 z_{19}^{2} z_{38}+z_{27}^{2} z_{38}-3 z_{17} z_{29} z_{38}-2 z_{18} z_{19} z_{39} \\
& -z_{26} z_{27} z_{39}+2 z_{17} z_{28} z_{39}+z_{16} z_{29} z_{39}+2 z_{19} z_{35} z_{39}+2 z_{25} z_{37} z_{39} \\
& -2 z_{15} z_{39}^{2}+2 z_{18} z_{25} z_{410}+z_{25} z_{26} z_{410}-3 z_{15} z_{28} z_{410}-z_{25} z_{35} z_{410}-z_{210} z_{26} z_{45} \\
& +z_{110} z_{28} z_{45}+z_{25} z_{310} z_{45}+2 z_{29} z_{36} z_{45}-2 z_{19} z_{38} z_{45}-2 z_{35} z_{39} z_{45} \\
& +2 z_{38} z_{45}^{2}+z_{19} z_{28} z_{46}-z_{26} z_{29} z_{46}+z_{25} z_{39} z_{46}-2 z_{28} z_{45} z_{46}-z_{18} z_{28} z_{47}
\end{aligned}
$$




$$
\begin{aligned}
& +z_{26} z_{28} z_{47}+2 z_{28} z_{35} z_{47}-3 z_{25} z_{38} z_{47}-2 z_{18} z_{19} z_{48}-z_{26} z_{27} z_{48}+z_{17} z_{28} z_{48} \\
& +2 z_{16} z_{29} z_{48}+z_{25} z_{37} z_{48}-2 z_{15} z_{39} z_{48}+2 z_{18} z_{45} z_{48}-2 z_{35} z_{45} z_{48} \\
& +2 z_{25} z_{46} z_{48}-2 z_{15} z_{48}^{2}+2 z_{18}^{2} z_{49}+z_{26}^{2} z_{49}-3 z_{16} z_{28} z_{49}-2 z_{18} z_{35} z_{49}+2 z_{35}^{2} z_{49} \\
& -3 z_{25} z_{36} z_{49}+6 z_{15} z_{38} z_{49}+z_{12} z_{210} z_{510}-z_{19} z_{23} z_{510}+z_{18} z_{24} z_{510}-z_{14} z_{28} z_{510} \\
& +z_{13} z_{29} z_{510}-2 z_{24} z_{35} z_{510}+2 z_{23} z_{45} z_{510}-z_{210} z_{24} z_{56}-z_{29} z_{34} z_{56}+2 z_{24} z_{39} z_{56} \\
& +z_{24} z_{48} z_{56}-3 z_{23} z_{49} z_{56}+z_{210} z_{23} z_{57}+z_{28} z_{34} z_{57}-3 z_{24} z_{38} z_{57}+z_{23} z_{39} z_{57} \\
& +2 z_{23} z_{48} z_{57}+z_{110} z_{24} z_{58}+z_{19} z_{34} z_{58}-z_{24} z_{37} z_{58}-z_{14} z_{39} z_{58}+z_{12} z_{410} z_{58} \\
& -z_{34} z_{45} z_{58}+z_{23} z_{47} z_{58}-z_{14} z_{48} z_{58}+2 z_{13} z_{49} z_{58}-z_{110} z_{23} z_{59}-z_{12} z_{310} z_{59} \\
& -z_{18} z_{34} z_{59}+z_{34} z_{35} z_{59}+z_{24} z_{36} z_{59}+2 z_{14} z_{38} z_{59}-z_{13} z_{39} z_{59}-z_{23} z_{46} z_{59} \\
& -z_{13} z_{48} z_{59}+2 z_{24} z_{25} z_{610}-2 z_{12} z_{29} z_{610}+2 z_{24} z_{28} z_{67}-2 z_{23} z_{29} z_{67} \\
& -2 z_{19} z_{24} z_{68}+z_{24} z_{27} z_{68}+z_{14} z_{29} z_{68}+z_{24} z_{45} z_{68}-3 z_{12} z_{49} z_{68}+2 z_{19} z_{23} z_{69} \\
& -z_{24} z_{26} z_{69}-z_{14} z_{28} z_{69}-z_{25} z_{34} z_{69}+2 z_{12} z_{39} z_{69}-z_{23} z_{45} z_{69}+z_{12} z_{48} z_{69} \\
& -2 z_{23} z_{25} z_{710}+2 z_{12} z_{28} z_{710}+2 z_{18} z_{24} z_{78}-z_{23} z_{27} z_{78}-z_{13} z_{29} z_{78}+z_{25} z_{34} z_{78} \\
& -z_{24} z_{35} z_{78}+z_{12} z_{39} z_{78}+2 z_{12} z_{48} z_{78}-2 z_{18} z_{23} z_{79}+z_{23} z_{26} z_{79} \\
& +z_{13} z_{28} z_{79}+z_{23} z_{35} z_{79}-3 z_{12} z_{38} z_{79}+z_{12} z_{19} z_{810} \\
& -3 z_{15} z_{24} z_{810}+z_{14} z_{25} z_{810}+z_{12} z_{27} z_{810}-2 z_{12} z_{45} z_{810}+z_{14} z_{18} z_{89} \\
& -z_{13} z_{19} z_{89}-z_{17} z_{23} z_{89}+z_{16} z_{24} z_{89}+2 z_{15} z_{34} z_{89} \\
& -z_{14} z_{35} z_{89}-z_{12} z_{37} z_{89}+z_{13} z_{45} z_{89}+z_{12} z_{46} z_{89}-z_{12} z_{18} z_{910} \\
& +3 z_{15} z_{23} z_{910}-z_{13} z_{25} z_{910}-z_{12} z_{26} z_{910}+2 z_{12} z_{35} z_{910} \text {, } \\
& \psi_{14}=2 z_{110} z_{18} z_{210}-2 z_{16} z_{210}^{2}+2 z_{110} z_{210} z_{26}-2 z_{110}^{2} z_{28}-2 z_{18} z_{19} z_{310} \\
& +z_{15} z_{210} z_{310}-z_{110} z_{25} z_{310}-z_{19} z_{26} z_{310}-z_{18} z_{27} z_{310}-2 z_{26} z_{27} z_{310} \\
& +3 z_{17} z_{28} z_{310}+3 z_{16} z_{29} z_{310}+2 z_{210} z_{27} z_{36} \\
& -2 z_{110} z_{29} z_{36}+z_{25} z_{310} z_{37}-z_{210} z_{35} z_{37}-z_{29} z_{36} z_{37}-z_{28} z_{37}^{2} \\
& +2 z_{110} z_{19} z_{38}-2 z_{17} z_{210} z_{38}+z_{27} z_{37} z_{38}-z_{15} z_{310} z_{39} \\
& +z_{110} z_{35} z_{39}+z_{19} z_{36} z_{39}+z_{18} z_{37} z_{39}+z_{26} z_{37} z_{39} \\
& -z_{17} z_{38} z_{39}-z_{16} z_{39}^{2}+2 z_{18}^{2} z_{410}+2 z_{18} z_{26} z_{410}+2 z_{26}^{2} z_{410} \\
& -6 z_{16} z_{28} z_{410}+z_{35}^{2} z_{410}-3 z_{25} z_{36} z_{410}+3 z_{15} z_{38} z_{410}-z_{310} z_{35} z_{45} \\
& +z_{210} z_{36} z_{45}-z_{110} z_{38} z_{45}-2 z_{210} z_{26} z_{46}+2 z_{110} z_{28} z_{46} \\
& +2 z_{25} z_{310} z_{46}+z_{29} z_{36} z_{46}-z_{19} z_{38} z_{46}-z_{35} z_{39} z_{46} \\
& +2 z_{38} z_{45} z_{46}-2 z_{28} z_{46}^{2}+3 z_{28} z_{36} z_{47}-z_{1} z_{38} z_{47} \\
& -2 z_{26} z_{38} z_{47}-z_{35} z_{38} z_{47}-2 z_{110} z_{18} z_{48}+2 z_{16} z_{210} z_{48} \\
& -2 z_{15} z_{310} z_{48}-z_{27} z_{36} z_{48}+z_{35} z_{37} z_{48}+z_{17} z_{38} z_{48}-2 z_{36} z_{45} z_{48}+2 z_{18} z_{46} z_{48} \\
& +2 z_{26} z_{46} z_{48}-2 z_{16} z_{48}^{2}-2 z_{18} z_{36} z_{49}-z_{26} z_{36} z_{49}+z_{35} z_{36} z_{49} \\
& +3 z_{16} z_{38} z_{49}+2 z_{13} z_{210} z_{510}-2 z_{110} z_{23} z_{510}-z_{34} z_{35} z_{510}-z_{24} z_{36} z_{510} \\
& +z_{14} z_{38} z_{510}+z_{23} z_{46} z_{510}-z_{13} z_{48} z_{510}+z_{24} z_{310} z_{56}-2 z_{210} z_{34} z_{56}+z_{34} z_{39} z_{56} \\
& -3 z_{23} z_{410} z_{56}+z_{34} z_{48} z_{56}+2 z_{23} z_{310} z_{57}-2 z_{34} z_{38} z_{57}-z_{14} z_{310} z_{58} \\
& +2 z_{110} z_{34} z_{58}-z_{34} z_{37} z_{58}+3 z_{13} z_{410} z_{58}-z_{34} z_{46} z_{58} \\
& -2 z_{13} z_{310} z_{59}+2 z_{34} z_{36} z_{59}-z_{12} z_{210} z_{610}+z_{19} z_{23} z_{610}+z_{18} z_{24} z_{610}
\end{aligned}
$$




$$
\begin{aligned}
& +z_{24} z_{26} z_{610}-2 z_{14} z_{28} z_{610}-z_{13} z_{29} z_{610}+z_{25} z_{34} z_{610} \\
& +z_{23} z_{45} z_{610}+z_{12} z_{48} z_{610}-z_{210} z_{23} z_{67} \\
& +3 z_{28} z_{34} z_{67}-z_{24} z_{38} z_{67}-z_{23} z_{39} z_{67} \\
& +2 z_{23} z_{48} z_{67}+z_{14} z_{210} z_{68}-z_{110} z_{24} z_{68}-z_{19} z_{34} z_{68}+z_{27} z_{34} z_{68} \\
& -2 z_{12} z_{410} z_{68}+z_{24} z_{46} z_{68}+z_{23} z_{47} z_{68}-z_{14} z_{48} z_{68}-z_{13} z_{49} z_{68} \\
& +z_{110} z_{23} z_{69}+z_{12} z_{310} z_{69}-z_{18} z_{34} z_{69}-2 z_{26} z_{34} z_{69}+z_{14} z_{38} z_{69} \\
& +z_{13} z_{39} z_{69}-2 z_{23} z_{46} z_{69}-2 z_{18} z_{23} z_{710}-z_{23} z_{26} z_{710} \\
& +3 z_{13} z_{28} z_{710}-z_{23} z_{35} z_{710}-z_{12} z_{38} z_{710}-z_{13} z_{210} z_{78}+z_{12} z_{310} z_{78} \\
& +2 z_{18} z_{34} z_{78}+z_{26} z_{34} z_{78}-z_{24} z_{36} z_{78}-z_{23} z_{37} z_{78}+2 z_{13} z_{48} z_{78} \\
& +2 z_{23} z_{36} z_{79}-2 z_{13} z_{38} z_{79}+z_{110} z_{12} z_{810}+z_{14} z_{18} z_{810} \\
& -z_{17} z_{23} z_{810}-2 z_{16} z_{24} z_{810}+z_{14} z_{26} z_{810}+z_{13} z_{27} z_{810}-z_{15} z_{34} z_{810} \\
& -z_{13} z_{45} z_{810}-z_{12} z_{46} z_{810}-z_{110} z_{13} z_{89} \\
& +3 z_{16} z_{34} z_{89}-z_{14} z_{36} z_{89}-z_{13} z_{37} z_{89}+2 z_{13} z_{46} z_{89}-z_{13} z_{18} z_{910} \\
& +3 z_{16} z_{23} z_{910}-2 z_{13} z_{26} z_{910}+z_{13} z_{35} z_{910}+z_{12} z_{36} z_{910} \\
& \psi_{15}=2 z_{110} z_{19} z_{210}-2 z_{17} z_{210}^{2}+2 z_{110} z_{210} z_{27}-2 z_{110}^{2} z_{29}-2 z_{19}^{2} z_{310} \\
& -2 z_{19} z_{27} z_{310}-2 z_{27}^{2} z_{310}+6 z_{17} z_{29} z_{310}+2 z_{210} z_{27} z_{37}-2 z_{110} z_{29} z_{37}-2 z_{29} z_{37}^{2} \\
& +2 z_{110} z_{19} z_{39}-2 z_{17} z_{210} z_{39}+2 z_{19} z_{37} z_{39}+2 z_{27} z_{37} z_{39}-2 z_{17} z_{39}^{2} \\
& +2 z_{18} z_{19} z_{410}+z_{15} z_{210} z_{410}-z_{110} z_{25} z_{410}+z_{19} z_{26} z_{410}+z_{18} z_{27} z_{410} \\
& +2 z_{26} z_{27} z_{410}-3 z_{17} z_{28} z_{410}-3 z_{16} z_{29} z_{410}-2 z_{25} z_{37} z_{410} \\
& +2 z_{15} z_{39} z_{410}+z_{35} z_{410} z_{45}-z_{310} z_{45}^{2} \\
& -z_{25} z_{410} z_{46}+z_{210} z_{45} z_{46}+z_{39} z_{45} z_{46}-z_{29} z_{46}^{2}-2 z_{210} z_{26} z_{47}+2 z_{110} z_{28} z_{47} \\
& +3 z_{25} z_{310} z_{47}-z_{210} z_{35} z_{47}+3 z_{29} z_{36} z_{47}+z_{28} z_{37} z_{47} \\
& -2 z_{19} z_{38} z_{47}-z_{27} z_{38} z_{47}-z_{26} z_{39} z_{47} \\
& -2 z_{35} z_{39} z_{47}+z_{38} z_{45} z_{47}-z_{28} z_{46} z_{47}+z_{15} z_{410} z_{48}-z_{110} z_{45} z_{48}-z_{37} z_{45} z_{48} \\
& +z_{19} z_{46} z_{48}+z_{27} z_{46} z_{48}+z_{18} z_{47} z_{48}-z_{17} z_{48}^{2}-2 z_{110} z_{18} z_{49}+2 z_{16} z_{210} z_{49} \\
& -3 z_{15} z_{310} z_{49}+z_{110} z_{35} z_{49}-z_{19} z_{36} z_{49}-2 z_{27} z_{36} z_{49}-z_{18} z_{37} z_{49}+2 z_{35} z_{37} z_{49} \\
& +3 z_{17} z_{38} z_{49}+z_{16} z_{39} z_{49}-z_{36} z_{45} z_{49}+z_{26} z_{46} z_{49}-z_{16} z_{48} z_{49}+2 z_{14} z_{210} z_{510} \\
& -2 z_{110} z_{24} z_{510}-z_{24} z_{37} z_{510}+z_{14} z_{39} z_{510}-z_{34} z_{45} z_{510}+z_{23} z_{47} z_{510} \\
& -z_{13} z_{49} z_{510}-2 z_{24} z_{410} z_{56}+2 z_{34} z_{49} z_{56}+3 z_{24} z_{310} z_{57}-2 z_{210} z_{34} z_{57} \\
& -z_{34} z_{39} z_{57}-z_{23} z_{410} z_{57}-z_{34} z_{48} z_{57} \\
& +2 z_{14} z_{410} z_{58}-2 z_{34} z_{47} z_{58}-3 z_{14} z_{310} z_{59}+2 z_{110} z_{34} z_{59} \\
& +z_{34} z_{37} z_{59}+z_{13} z_{410} z_{59}+z_{34} z_{46} z_{59} \\
& +2 z_{19} z_{24} z_{610}+z_{24} z_{27} z_{610}-3 z_{14} z_{29} z_{610}+z_{24} z_{45} z_{610} \\
& +z_{12} z_{49} z_{610}-z_{210} z_{24} z_{67}+3 z_{29} z_{34} z_{67} \\
& -2 z_{24} z_{39} z_{67}+z_{24} z_{48} z_{67}+z_{23} z_{49} z_{67}+2 z_{24} z_{47} z_{68}-2 z_{14} z_{49} z_{68}+z_{14} z_{210} z_{69} \\
& -2 z_{19} z_{34} z_{69}-z_{27} z_{34} z_{69}+2 z_{14} z_{39} z_{69}-z_{12} z_{410} z_{69} \\
& -z_{24} z_{46} z_{69}-z_{23} z_{47} z_{69}-z_{12} z_{210} z_{710} \\
& -z_{19} z_{23} z_{710}-z_{18} z_{24} z_{710}-z_{23} z_{27} z_{710}+z_{14} z_{28} z_{710}
\end{aligned}
$$




$$
\begin{aligned}
& +2 z_{13} z_{29} z_{710}+z_{25} z_{34} z_{710}-z_{24} z_{35} z_{710} \\
& -z_{12} z_{39} z_{710}-z_{110} z_{24} z_{78}+z_{19} z_{34} z_{78} \\
& +2 z_{27} z_{34} z_{78}-2 z_{24} z_{37} z_{78}-z_{12} z_{410} z_{78} \\
& +z_{14} z_{48} z_{78}+z_{13} z_{49} z_{78}-z_{13} z_{210} z_{79}+z_{110} z_{23} z_{79} \\
& +2 z_{12} z_{310} z_{79}+z_{18} z_{34} z_{79}-z_{26} z_{34} z_{79} \\
& +z_{24} z_{36} z_{79}+z_{23} z_{37} z_{79}-z_{14} z_{38} z_{79}-z_{13} z_{39} z_{79}+z_{14} z_{19} z_{810}-3 z_{17} z_{24} z_{810} \\
& +2 z_{14} z_{27} z_{810}-z_{14} z_{45} z_{810}-z_{12} z_{47} z_{810} \\
& -z_{110} z_{14} z_{89}+3 z_{17} z_{34} z_{89}-2 z_{14} z_{37} z_{89} \\
& +z_{14} z_{46} z_{89}+z_{13} z_{47} z_{89}+z_{110} z_{12} z_{910} \\
& -z_{13} z_{19} z_{910}+2 z_{17} z_{23} z_{910}+z_{16} z_{24} z_{910} \\
& -z_{14} z_{26} z_{910}-z_{13} z_{27} z_{910}-z_{15} z_{34} z_{910}+z_{14} z_{35} z_{910}+z_{12} z_{37} z_{910} \text {, } \\
& \psi_{23}=-z_{15} z_{210} z_{510}+z_{110} z_{25} z_{510}+z_{19} z_{35} z_{510}-z_{27} z_{35} z_{510} \\
& -z_{18} z_{45} z_{510}+z_{26} z_{45} z_{510}+z_{12} z_{510}^{2}+z_{19} z_{210} z_{56} \\
& -2 z_{210} z_{27} z_{56}+z_{110} z_{29} z_{56}+z_{29} z_{37} z_{56}-2 z_{19} z_{39} z_{56} \\
& +z_{27} z_{39} z_{56}+2 z_{25} z_{410} z_{56}-z_{28} z_{47} z_{56}+z_{45} z_{48} z_{56} \\
& +2 z_{18} z_{49} z_{56}-z_{26} z_{49} z_{56}-z_{35} z_{49} z_{56}+z_{24} z_{510} z_{56} \\
& -z_{18} z_{210} z_{57}+2 z_{210} z_{26} z_{57}-z_{110} z_{28} z_{57}-2 z_{25} z_{310} z_{57} \\
& -z_{29} z_{36} z_{57}+2 z_{19} z_{38} z_{57}-z_{27} z_{38} z_{57}+z_{35} z_{39} z_{57} \\
& -z_{38} z_{45} z_{57}+z_{28} z_{46} z_{57}-2 z_{18} z_{48} z_{57}+z_{26} z_{48} z_{57} \\
& -z_{23} z_{510} z_{57}-2 z_{110} z_{19} z_{58}+z_{17} z_{210} z_{58}+z_{110} z_{27} z_{58} \\
& +z_{19} z_{37} z_{58}-2 z_{27} z_{37} z_{58}+z_{17} z_{39} z_{58}-2 z_{15} z_{410} z_{58} \\
& -z_{45} z_{46} z_{58}-z_{18} z_{47} z_{58}+2 z_{26} z_{47} z_{58}+z_{35} z_{47} z_{58} \\
& -z_{16} z_{49} z_{58}-z_{14} z_{510} z_{58}+2 z_{34} z_{57} z_{58}+2 z_{110} z_{18} z_{59} \\
& -z_{16} z_{210} z_{59}-z_{110} z_{26} z_{59}+2 z_{15} z_{310} z_{59}-z_{19} z_{36} z_{59} \\
& +2 z_{27} z_{36} z_{59}-z_{35} z_{37} z_{59}-z_{17} z_{38} z_{59}+z_{36} z_{45} z_{59} \\
& +z_{18} z_{46} z_{59}-2 z_{26} z_{46} z_{59}+z_{16} z_{48} z_{59}+z_{13} z_{510} z_{59} \\
& -2 z_{34} z_{56} z_{59}-2 z_{19} z_{25} z_{610}+z_{25} z_{27} z_{610}+z_{15} z_{29} z_{610} \\
& -z_{25} z_{45} z_{610}-3 z_{12} z_{59} z_{610}-z_{19} z_{28} z_{67}+z_{27} z_{28} z_{67} \\
& +z_{18} z_{29} z_{67}-z_{26} z_{29} z_{67}+z_{25} z_{39} z_{67}-z_{25} z_{48} z_{67}+z_{24} z_{58} z_{67} \\
& -z_{23} z_{59} z_{67}+2 z_{19}^{2} z_{68}-2 z_{19} z_{27} z_{68}+2 z_{27}^{2} z_{68}-2 z_{17} z_{29} z_{68} \\
& +z_{45}^{2} z_{68}-3 z_{25} z_{47} z_{68}+3 z_{15} z_{49} z_{68}-3 z_{24} z_{57} z_{68} \\
& +3 z_{14} z_{59} z_{68}-2 z_{18} z_{19} z_{69}+2 z_{19} z_{26} z_{69}-2 z_{26} z_{27} z_{69} \\
& +z_{17} z_{28} z_{69}+z_{16} z_{29} z_{69}+z_{25} z_{37} z_{69}-2 z_{15} z_{39} z_{69} \\
& -z_{35} z_{45} z_{69}+2 z_{25} z_{46} z_{69}-z_{15} z_{48} z_{69}+z_{24} z_{56} z_{69} \\
& +2 z_{23} z_{57} z_{69}-2 z_{14} z_{58} z_{69}-z_{13} z_{59} z_{69}+2 z_{12} z_{69}^{2} \\
& +2 z_{18} z_{25} z_{710}-z_{25} z_{26} z_{710}-z_{15} z_{28} z_{710}+z_{25} z_{35} z_{710} \\
& +3 z_{12} z_{58} z_{710}-2 z_{18} z_{19} z_{78}+2 z_{18} z_{27} z_{78}-2 z_{26} z_{27} z_{78} \\
& +z_{17} z_{28} z_{78}+z_{16} z_{29} z_{78}+2 z_{25} z_{37} z_{78}-z_{15} z_{39} z_{78}
\end{aligned}
$$




$$
\begin{aligned}
& -z_{35} z_{45} z_{78}+z_{25} z_{46} z_{78}-2 z_{15} z_{48} z_{78}+2 z_{24} z_{56} z_{78} \\
& +z_{23} z_{57} z_{78}-z_{14} z_{58} z_{78}-2 z_{13} z_{59} z_{78}+2 z_{12} z_{69} z_{78} \\
& +2 z_{12} z_{78}^{2}+2 z_{18}^{2} z_{79}-2 z_{18} z_{26} z_{79}+2 z_{26}^{2} z_{79}-2 z_{16} z_{28} z_{79} \\
& +z_{35}^{2} z_{79}-3 z_{25} z_{36} z_{79}+3 z_{15} z_{38} z_{79}-3 z_{23} z_{56} z_{79} \\
& +3 z_{13} z_{58} z_{79}-6 z_{12} z_{68} z_{79}+z_{15} z_{19} z_{810}+z_{17} z_{25} z_{810} \\
& -2 z_{15} z_{27} z_{810}+z_{15} z_{45} z_{810}+3 z_{12} z_{57} z_{810}+z_{17} z_{18} z_{89} \\
& -z_{16} z_{19} z_{89}-z_{17} z_{26} z_{89}+z_{16} z_{27} z_{89}+z_{15} z_{37} z_{89} \\
& -z_{15} z_{46} z_{89}+z_{14} z_{56} z_{89}-z_{13} z_{57} z_{89}-2 z_{12} z_{67} z_{89} \\
& -z_{15} z_{18} z_{910}-z_{16} z_{25} z_{910}+2 z_{15} z_{26} z_{910}-z_{15} z_{35} z_{910}-3 z_{12} z_{56} z_{910} \text {, } \\
& \psi_{24}=2 z_{110} z_{18} z_{510}-2 z_{16} z_{210} z_{510}+z_{15} z_{310} z_{510}+2 z_{110} z_{35} z_{510} \\
& +z_{27} z_{36} z_{510}-2 z_{35} z_{37} z_{510}-z_{17} z_{38} z_{510}+2 z_{36} z_{45} z_{510} \\
& -z_{26} z_{46} z_{510}+z_{16} z_{48} z_{510}+2 z_{13} z_{510}^{2}+2 z_{110} z_{210} z_{56} \\
& -z_{19} z_{310} z_{56}-z_{27} z_{310} z_{56}-z_{210} z_{37} z_{56}-z_{110} z_{39} z_{56} \\
& +2 z_{37} z_{39} z_{56}+2 z_{18} z_{410} z_{56}+z_{26} z_{410} z_{56}+z_{35} z_{410} z_{56} \\
& -z_{38} z_{47} z_{56}+z_{46} z_{48} z_{56}-2 z_{36} z_{49} z_{56}-z_{34} z_{510} z_{56}-z_{18} z_{310} z_{57} \\
& -z_{310} z_{35} z_{57}+z_{210} z_{36} z_{57}+z_{110} z_{38} z_{57}-z_{37} z_{38} z_{57} \\
& +z_{36} z_{48} z_{57}-2 z_{110}^{2} z_{58}+2 z_{17} z_{310} z_{58}+2 z_{110} z_{37} z_{58}-2 z_{37}^{2} z_{58} \\
& -3 z_{16} z_{410} z_{58}-z_{46}^{2} z_{58}+3 z_{36} z_{47} z_{58}+z_{16} z_{310} z_{59} \\
& -2 z_{110} z_{36} z_{59}+z_{36} z_{37} z_{59}-z_{36} z_{46} z_{59}-2 z_{18} z_{19} z_{610} \\
& -z_{110} z_{25} z_{610}-z_{26} z_{27} z_{610}+z_{17} z_{28} z_{610}+2 z_{16} z_{29} z_{610}-z_{19} z_{35} z_{610} \\
& +2 z_{25} z_{37} z_{610}-z_{15} z_{39} z_{610}-z_{18} z_{45} z_{610}-2 z_{35} z_{45} z_{610} \\
& +z_{25} z_{46} z_{610}-z_{15} z_{48} z_{610}-z_{12} z_{510} z_{610}+2 z_{24} z_{56} z_{610}+z_{23} z_{57} z_{610} \\
& -3 z_{14} z_{58} z_{610}-3 z_{13} z_{59} z_{610}+z_{210} z_{26} z_{67}-2 z_{110} z_{28} z_{67}-z_{25} z_{310} z_{67} \\
& -2 z_{29} z_{36} z_{67}+z_{28} z_{37} z_{67}+z_{19} z_{38} z_{67}+z_{18} z_{39} z_{67} \\
& +2 z_{35} z_{39} z_{67}-z_{38} z_{45} z_{67}+z_{28} z_{46} z_{67}-2 z_{18} z_{48} z_{67}-z_{35} z_{48} z_{67} \\
& -2 z_{23} z_{510} z_{67}+3 z_{34} z_{58} z_{67}+2 z_{110} z_{19} z_{68}-z_{17} z_{210} z_{68} \\
& -z_{110} z_{27} z_{68}-z_{19} z_{37} z_{68}+2 z_{27} z_{37} z_{68}-z_{17} z_{39} z_{68}+z_{15} z_{410} z_{68} \\
& +z_{45} z_{46} z_{68}-z_{18} z_{47} z_{68}-z_{26} z_{47} z_{68}-2 z_{35} z_{47} z_{68}+2 z_{16} z_{49} z_{68} \\
& +2 z_{14} z_{510} z_{68}-z_{34} z_{57} z_{68}-2 z_{24} z_{67} z_{68}+z_{110} z_{26} z_{69}+z_{19} z_{36} z_{69} \\
& -z_{26} z_{37} z_{69}-z_{16} z_{39} z_{69}+z_{18} z_{46} z_{69}+z_{35} z_{46} z_{69}-z_{34} z_{56} z_{69} \\
& +z_{12} z_{610} z_{69}+z_{23} z_{67} z_{69}+z_{14} z_{68} z_{69}+z_{13} z_{69}^{2} \\
& +2 z_{18}^{2} z_{710}+z_{26}^{2} z_{710}-3 z_{16} z_{28} z_{710}+2 z_{18} z_{35} z_{710}+2 z_{35}^{2} z_{710} \\
& -3 z_{25} z_{36} z_{710}+2 z_{15} z_{38} z_{710}-3 z_{23} z_{56} z_{710}+6 z_{13} z_{58} z_{710} \\
& -3 z_{12} z_{68} z_{710}-2 z_{110} z_{18} z_{78}+z_{16} z_{210} z_{78}-z_{15} z_{310} z_{78} \\
& -2 z_{27} z_{36} z_{78}+2 z_{18} z_{37} z_{78}+2 z_{35} z_{37} z_{78}+z_{17} z_{38} z_{78} \\
& -z_{36} z_{45} z_{78}+z_{26} z_{46} z_{78}-2 z_{16} z_{48} z_{78}-2 z_{13} z_{510} z_{78} \\
& +2 z_{34} z_{56} z_{78}+2 z_{12} z_{610} z_{78}+z_{23} z_{67} z_{78}-z_{14} z_{68} z_{78} \\
& +2 z_{13} z_{78}^{2}-2 z_{18} z_{36} z_{79}+z_{26} z_{36} z_{79}-z_{35} z_{36} z_{79}+z_{16} z_{38} z_{79}
\end{aligned}
$$




$$
\begin{aligned}
& -3 z_{13} z_{68} z_{79}+z_{110} z_{15} z_{810}+z_{17} z_{18} z_{810}-z_{16} z_{27} z_{810} \\
& +z_{17} z_{35} z_{810}-z_{15} z_{37} z_{810}+z_{16} z_{45} z_{810}+z_{14} z_{56} z_{810} \\
& +2 z_{13} z_{57} z_{810}+z_{12} z_{67} z_{810}-z_{110} z_{16} z_{89}-z_{17} z_{36} z_{89} \\
& +2 z_{16} z_{37} z_{89}-z_{16} z_{46} z_{89}-3 z_{13} z_{67} z_{89}-z_{16} z_{18} z_{910} \\
& +z_{16} z_{26} z_{910}-2 z_{16} z_{35} z_{910}+z_{15} z_{36} z_{910}-3 z_{13} z_{56} z_{910} \text {, } \\
& \psi_{25}=2 z_{110} z_{19} z_{510}-2 z_{17} z_{210} z_{510}+z_{27} z_{37} z_{510}-z_{17} z_{39} z_{510} \\
& +z_{15} z_{410} z_{510}+2 z_{110} z_{45} z_{510}+2 z_{45} z_{46} z_{510}-z_{26} z_{47} z_{510} \\
& -2 z_{35} z_{47} z_{510}+z_{16} z_{49} z_{510}+2 z_{14} z_{510}^{2}+z_{19} z_{410} z_{56} \\
& +z_{410} z_{45} z_{56}-z_{210} z_{47} z_{56}+z_{39} z_{47} z_{56}-z_{110} z_{49} z_{56}-z_{46} z_{49} z_{56} \\
& +2 z_{110} z_{210} z_{57}-2 z_{19} z_{310} z_{57}-z_{27} z_{310} z_{57}+z_{37} z_{39} z_{57} \\
& +z_{18} z_{410} z_{57}+z_{26} z_{410} z_{57}-z_{310} z_{45} z_{57}+z_{210} z_{46} z_{57} \\
& -2 z_{38} z_{47} z_{57}+z_{110} z_{48} z_{57}+2 z_{46} z_{48} z_{57}-z_{36} z_{49} z_{57}-z_{34} z_{510} z_{57} \\
& -z_{17} z_{410} z_{58}+2 z_{110} z_{47} z_{58}-z_{37} z_{47} z_{58}+z_{46} z_{47} z_{58}-2 z_{110}^{2} z_{59} \\
& +3 z_{17} z_{310} z_{59}-z_{37}^{2} z_{59}-2 z_{16} z_{410} z_{59}-2 z_{110} z_{46} z_{59}-2 z_{46}^{2} z_{59} \\
& +3 z_{36} z_{47} z_{59}-2 z_{19}^{2} z_{610}-z_{27}^{2} z_{610}+3 z_{17} z_{29} z_{610}-2 z_{19} z_{45} z_{610} \\
& -2 z_{45}^{2} z_{610}+3 z_{25} z_{47} z_{610}-2 z_{15} z_{49} z_{610}+3 z_{24} z_{57} z_{610} \\
& -6 z_{14} z_{59} z_{610}+z_{210} z_{27} z_{67}-2 z_{110} z_{29} z_{67}-z_{29} z_{37} z_{67} \\
& +2 z_{19} z_{39} z_{67}-z_{25} z_{410} z_{67}+z_{39} z_{45} z_{67}-z_{29} z_{46} z_{67} \\
& +2 z_{28} z_{47} z_{67}-z_{19} z_{48} z_{67}-2 z_{45} z_{48} z_{67}-z_{18} z_{49} z_{67}+z_{35} z_{49} z_{67} \\
& -2 z_{24} z_{510} z_{67}+3 z_{34} z_{59} z_{67}-2 z_{19} z_{47} z_{68}+z_{27} z_{47} z_{68} \\
& -z_{45} z_{47} z_{68}+z_{17} z_{49} z_{68}+2 z_{110} z_{19} z_{69}-z_{17} z_{210} z_{69} \\
& +z_{27} z_{37} z_{69}-2 z_{17} z_{39} z_{69}+z_{15} z_{410} z_{69}+2 z_{19} z_{46} z_{69} \\
& +2 z_{45} z_{46} z_{69}-2 z_{26} z_{47} z_{69}-z_{35} z_{47} z_{69}+z_{16} z_{49} z_{69} \\
& +2 z_{14} z_{510} z_{69}-2 z_{34} z_{57} z_{69}-z_{24} z_{67} z_{69}+2 z_{14} z_{69}^{2} \\
& +2 z_{18} z_{19} z_{710}-z_{110} z_{25} z_{710}+z_{26} z_{27} z_{710}-2 z_{17} z_{28} z_{710} \\
& -z_{16} z_{29} z_{710}+z_{19} z_{35} z_{710}-z_{25} z_{37} z_{710}+z_{15} z_{39} z_{710}+z_{18} z_{45} z_{710} \\
& +2 z_{35} z_{45} z_{710}-2 z_{25} z_{46} z_{710}+z_{15} z_{48} z_{710}-z_{12} z_{510} z_{710}-z_{24} z_{56} z_{710} \\
& -2 z_{23} z_{57} z_{710}+3 z_{14} z_{58} z_{710}+3 z_{13} z_{59} z_{710}-2 z_{12} z_{69} z_{710} \\
& -z_{110} z_{27} z_{78}+z_{19} z_{37} z_{78}+z_{37} z_{45} z_{78}-z_{27} z_{46} z_{78}+z_{18} z_{47} z_{78} \\
& -z_{17} z_{48} z_{78}+z_{34} z_{57} z_{78}-z_{24} z_{67} z_{78}-z_{12} z_{710} z_{78}+z_{14} z_{78}^{2} \\
& -2 z_{110} z_{18} z_{79}+z_{16} z_{210} z_{79}+z_{110} z_{26} z_{79}-z_{15} z_{310} z_{79} \\
& -z_{19} z_{36} z_{79}-z_{27} z_{36} z_{79}+z_{35} z_{37} z_{79}+2 z_{17} z_{38} z_{79}-2 z_{36} z_{45} z_{79} \\
& -z_{18} z_{46} z_{79}+2 z_{26} z_{46} z_{79}-z_{16} z_{48} z_{79}-2 z_{13} z_{510} z_{79} \\
& +z_{34} z_{56} z_{79}+3 z_{12} z_{610} z_{79}+2 z_{23} z_{67} z_{79}-3 z_{14} z_{68} z_{79} \\
& -z_{13} z_{69} z_{79}+z_{13} z_{78} z_{79}+z_{17} z_{19} z_{810}-z_{17} z_{27} z_{810} \\
& +2 z_{17} z_{45} z_{810}-z_{15} z_{47} z_{810}+3 z_{14} z_{57} z_{810}-z_{110} z_{17} z_{89} \\
& +z_{17} z_{37} z_{89}-2 z_{17} z_{46} z_{89}+z_{16} z_{47} z_{89}-3 z_{14} z_{67} z_{89}+z_{110} z_{15} z_{910} \\
& -z_{16} z_{19} z_{910}+z_{17} z_{26} z_{910}-z_{17} z_{35} z_{910}-z_{16} z_{45} z_{910}
\end{aligned}
$$




$$
\begin{aligned}
& +z_{15} z_{46} z_{910}-2 z_{14} z_{56} z_{910}-z_{13} z_{57} z_{910}+z_{12} z_{67} z_{910} \\
& \psi_{34}=-2 z_{210} z_{26} z_{510}+2 z_{110} z_{28} z_{510}+z_{25} z_{310} z_{510}+2 z_{210} z_{35} z_{510} \\
& +z_{29} z_{36} z_{510}-z_{19} z_{38} z_{510}-2 z_{35} z_{39} z_{510}+2 z_{38} z_{45} z_{510}-z_{28} z_{46} z_{510} \\
& +z_{18} z_{48} z_{510}+2 z_{23} z_{510}^{2}+2 z_{210}^{2} z_{56}-2 z_{29} z_{310} z_{56}-2 z_{210} z_{39} z_{56} \\
& +2 z_{39}^{2} z_{56}+3 z_{28} z_{410} z_{56}+z_{48}^{2} z_{56}-3 z_{38} z_{49} z_{56}-z_{28} z_{310} z_{57} \\
& +2 z_{210} z_{38} z_{57}-z_{38} z_{39} z_{57}+z_{38} z_{48} z_{57}-2 z_{110} z_{210} z_{58}+z_{19} z_{310} z_{58} \\
& +z_{27} z_{310} z_{58}+z_{210} z_{37} z_{58}+z_{110} z_{39} z_{58}-2 z_{37} z_{39} z_{58}-z_{18} z_{410} z_{58} \\
& -2 z_{26} z_{410} z_{58}+z_{35} z_{410} z_{58}+2 z_{38} z_{47} z_{58}-z_{46} z_{48} z_{58}+z_{36} z_{49} z_{58} \\
& -z_{34} z_{510} z_{58}+z_{26} z_{310} z_{59}-z_{310} z_{35} z_{59}-z_{210} z_{36} z_{59}-z_{110} z_{38} z_{59} \\
& +z_{36} z_{39} z_{59}-z_{38} z_{46} z_{59}-z_{210} z_{25} z_{610}-z_{19} z_{28} z_{610}+z_{26} z_{29} z_{610} \\
& -z_{29} z_{35} z_{610}+z_{25} z_{39} z_{610}-z_{28} z_{45} z_{610}-z_{24} z_{58} z_{610}-2 z_{23} z_{59} z_{610} \\
& -z_{210} z_{28} z_{67}-z_{29} z_{38} z_{67}+2 z_{28} z_{39} z_{67}-z_{28} z_{48} z_{67}+z_{19} z_{210} z_{68} \\
& -2 z_{210} z_{27} z_{68}+z_{110} z_{29} z_{68}+z_{29} z_{37} z_{68}-2 z_{19} z_{39} z_{68}+z_{27} z_{39} z_{68} \\
& +z_{25} z_{410} z_{68}-2 z_{28} z_{47} z_{68}+z_{45} z_{48} z_{68}+z_{18} z_{49} z_{68}+z_{26} z_{49} z_{68} \\
& -2 z_{35} z_{49} z_{68}+2 z_{24} z_{510} z_{68}-z_{34} z_{59} z_{68}+2 z_{210} z_{26} z_{69}-z_{110} z_{28} z_{69} \\
& -z_{25} z_{310} z_{69}-z_{29} z_{36} z_{69}+2 z_{19} z_{38} z_{69}-2 z_{26} z_{39} z_{69}+2 z_{35} z_{39} z_{69} \\
& -z_{38} z_{45} z_{69}+2 z_{28} z_{46} z_{69}-z_{18} z_{48} z_{69}-2 z_{23} z_{510} z_{69} \\
& +2 z_{34} z_{58} z_{69}-z_{24} z_{68} z_{69}+2 z_{23} z_{69}^{2}+z_{18} z_{28} z_{710}-z_{26} z_{28} z_{710} \\
& +2 z_{28} z_{35} z_{710}-z_{25} z_{38} z_{710}+3 z_{23} z_{58} z_{710}-z_{18} z_{210} z_{78}+z_{28} z_{37} z_{78} \\
& -z_{27} z_{38} z_{78}+z_{18} z_{39} z_{78}-z_{26} z_{48} z_{78}+z_{35} z_{48} z_{78}-z_{34} z_{58} z_{78} \\
& +z_{24} z_{68} z_{78}+z_{23} z_{78}^{2}-z_{28} z_{36} z_{79}-z_{18} z_{38} z_{79}+2 z_{26} z_{38} z_{79} \\
& -z_{35} z_{38} z_{79}-3 z_{23} z_{68} z_{79}-z_{18} z_{19} z_{810}+z_{15} z_{210} z_{810}-2 z_{26} z_{27} z_{810} \\
& +2 z_{17} z_{28} z_{810}+z_{16} z_{29} z_{810}+z_{27} z_{35} z_{810}+z_{25} z_{37} z_{810} \\
& -2 z_{15} z_{39} z_{810}+z_{26} z_{45} z_{810}-2 z_{35} z_{45} z_{810}+z_{25} z_{46} z_{810}-z_{15} z_{48} z_{810} \\
& -z_{12} z_{510} z_{810}+3 z_{24} z_{56} z_{810}+3 z_{23} z_{57} z_{810}-2 z_{14} z_{58} z_{810}-z_{13} z_{59} z_{810} \\
& +2 z_{12} z_{69} z_{810}+z_{12} z_{78} z_{810}+z_{110} z_{18} z_{89}-2 z_{16} z_{210} z_{89}+z_{15} z_{310} z_{89} \\
& +z_{27} z_{36} z_{89}+z_{26} z_{37} z_{89}-2 z_{35} z_{37} z_{89}-2 z_{17} z_{38} z_{89}+z_{16} z_{39} z_{89} \\
& +z_{36} z_{45} z_{89}-2 z_{26} z_{46} z_{89}+z_{35} z_{46} z_{89}+z_{16} z_{48} z_{89}+2 z_{13} z_{510} z_{89} \\
& -3 z_{34} z_{56} z_{89}-z_{12} z_{610} z_{89}-3 z_{23} z_{67} z_{89}+2 z_{14} z_{68} z_{89}-z_{13} z_{69} z_{89} \\
& -z_{13} z_{78} z_{89}+z_{18}^{2} z_{910}+2 z_{26}^{2} z_{910}-3 z_{16} z_{28} z_{910}-2 z_{26} z_{35} z_{910}+2 z_{35}^{2} z_{910} \\
& -2 z_{25} z_{36} z_{910}+3 z_{15} z_{38} z_{910}-6 z_{23} z_{56} z_{910}+3 z_{13} z_{58} z_{910}-3 z_{12} z_{68} z_{910} \text {, } \\
& \psi_{35}=-2 z_{210} z_{27} z_{510}+2 z_{110} z_{29} z_{510}+z_{29} z_{37} z_{510}-z_{19} z_{39} z_{510}+z_{25} z_{410} z_{510} \\
& +2 z_{210} z_{45} z_{510}-z_{28} z_{47} z_{510}+2 z_{45} z_{48} z_{510}+z_{18} z_{49} z_{510} \\
& -2 z_{35} z_{49} z_{510}+2 z_{24} z_{510}^{2}+z_{29} z_{410} z_{56}-2 z_{210} z_{49} z_{56}+z_{39} z_{49} z_{56}-z_{48} z_{49} z_{56} \\
& +2 z_{210}^{2} z_{57}-3 z_{29} z_{310} z_{57}+z_{39}^{2} z_{57}+2 z_{28} z_{410} z_{57}+2 z_{210} z_{48} z_{57} \\
& +2 z_{48}^{2} z_{57}-3 z_{38} z_{49} z_{57}-z_{27} z_{410} z_{58}+z_{410} z_{45} z_{58}+z_{210} z_{47} z_{58} \\
& +z_{47} z_{48} z_{58}+z_{110} z_{49} z_{58}-z_{37} z_{49} z_{58}-2 z_{110} z_{210} z_{59}+z_{19} z_{310} z_{59}
\end{aligned}
$$




$$
\begin{aligned}
& +2 z_{27} z_{310} z_{59}-z_{37} z_{39} z_{59}-z_{18} z_{410} z_{59}-z_{26} z_{410} z_{59}-z_{310} z_{45} z_{59} \\
& -z_{210} z_{46} z_{59}+z_{38} z_{47} z_{59}-z_{110} z_{48} z_{59}-2 z_{46} z_{48} z_{59}+2 z_{36} z_{49} z_{59} \\
& -z_{34} z_{510} z_{59}-z_{19} z_{29} z_{610}+z_{27} z_{29} z_{610}-2 z_{29} z_{45} z_{610}+z_{25} z_{49} z_{610} \\
& -3 z_{24} z_{59} z_{610}-z_{210} z_{29} z_{67}+z_{29} z_{39} z_{67}-2 z_{29} z_{48} z_{67}+z_{28} z_{49} z_{67} \\
& -z_{29} z_{47} z_{68}-z_{19} z_{49} z_{68}+2 z_{27} z_{49} z_{68}-z_{45} z_{49} z_{68}+z_{19} z_{210} z_{69} \\
& -z_{27} z_{39} z_{69}+z_{39} z_{45} z_{69}+z_{29} z_{46} z_{69}+z_{19} z_{48} z_{69}-z_{26} z_{49} z_{69} \\
& +z_{34} z_{59} z_{69}+z_{24} z_{69}^{2}-z_{210} z_{25} z_{710}-z_{27} z_{28} z_{710}+z_{18} z_{29} z_{710} \\
& +z_{29} z_{35} z_{710}+z_{28} z_{45} z_{710}-z_{25} z_{48} z_{710}+2 z_{24} z_{58} z_{710}+z_{23} z_{59} z_{710} \\
& -2 z_{210} z_{27} z_{78}+z_{110} z_{29} z_{78}+2 z_{29} z_{37} z_{78}-z_{19} z_{39} z_{78}+z_{25} z_{410} z_{78} \\
& -z_{28} z_{47} z_{78}-2 z_{27} z_{48} z_{78}+2 z_{45} z_{48} z_{78}+2 z_{18} z_{49} z_{78}-z_{35} z_{49} z_{78} \\
& +2 z_{24} z_{510} z_{78}-2 z_{34} z_{59} z_{78}+2 z_{24} z_{78}^{2}-z_{18} z_{210} z_{79}+2 z_{210} z_{26} z_{79} \\
& -z_{110} z_{28} z_{79}-z_{25} z_{310} z_{79}-2 z_{29} z_{36} z_{79}+z_{19} z_{38} z_{79}+z_{27} z_{38} z_{79}+z_{35} z_{39} z_{79} \\
& -2 z_{38} z_{45} z_{79}+z_{28} z_{46} z_{79}-2 z_{18} z_{48} z_{79}+z_{26} z_{48} z_{79}-2 z_{23} z_{510} z_{79} \\
& +z_{34} z_{58} z_{79}-3 z_{24} z_{68} z_{79}+z_{23} z_{69} z_{79}-z_{23} z_{78} z_{79}-z_{19}^{2} z_{810}-2 z_{27}^{2} z_{810} \\
& +3 z_{17} z_{29} z_{810}+2 z_{27} z_{45} z_{810}-2 z_{45}^{2} z_{810}+2 z_{25} z_{47} z_{810}-3 z_{15} z_{49} z_{810} \\
& +6 z_{24} z_{57} z_{810}-3 z_{14} z_{59} z_{810}+3 z_{12} z_{79} z_{810}+z_{110} z_{19} z_{89}-2 z_{17} z_{210} z_{89} \\
& +2 z_{27} z_{37} z_{89}-z_{17} z_{39} z_{89}+z_{15} z_{410} z_{89}-z_{37} z_{45} z_{89}-z_{27} z_{46} z_{89} \\
& +2 z_{45} z_{46} z_{89}-z_{26} z_{47} z_{89}-z_{35} z_{47} z_{89}-z_{17} z_{48} z_{89}+2 z_{16} z_{49} z_{89} \\
& +2 z_{14} z_{510} z_{89}-3 z_{34} z_{57} z_{89}-3 z_{24} z_{67} z_{89}+z_{14} z_{69} z_{89}-z_{12} z_{710} z_{89} \\
& +z_{14} z_{78} z_{89}-2 z_{13} z_{79} z_{89}+z_{18} z_{19} z_{910}+z_{15} z_{210} z_{910}+2 z_{26} z_{27} z_{910} \\
& -z_{17} z_{28} z_{910}-2 z_{16} z_{29} z_{910}-z_{27} z_{35} z_{910}-z_{25} z_{37} z_{910}+z_{15} z_{39} z_{910} \\
& -z_{26} z_{45} z_{910}+2 z_{35} z_{45} z_{910}-z_{25} z_{46} z_{910}+2 z_{15} z_{48} z_{910} \\
& -z_{12} z_{510} z_{910}-3 z_{24} z_{56} z_{910}-3 z_{23} z_{57} z_{910}+z_{14} z_{58} z_{910} \\
& +2 z_{13} z_{59} z_{910}-z_{12} z_{69} z_{910}-2 z_{12} z_{78} z_{910} \\
& \psi_{45}=-z_{210} z_{37} z_{510}+z_{110} z_{39} z_{510}-z_{35} z_{410} z_{510}+z_{310} z_{45} z_{510} \\
& +z_{210} z_{46} z_{510}-z_{110} z_{48} z_{510}+z_{34} z_{510}^{2}-z_{210} z_{410} z_{56}+2 z_{39} z_{410} z_{56} \\
& -z_{410} z_{48} z_{56}-z_{310} z_{49} z_{56}+z_{210} z_{310} z_{57}-z_{310} z_{39} z_{57}-z_{38} z_{410} z_{57} \\
& +2 z_{310} z_{48} z_{57}+z_{110} z_{410} z_{58}-2 z_{37} z_{410} z_{58}+z_{410} z_{46} z_{58}+z_{310} z_{47} z_{58} \\
& -z_{110} z_{310} z_{59}+z_{310} z_{37} z_{59}+z_{36} z_{410} z_{59}-2 z_{310} z_{46} z_{59}-z_{210} z_{27} z_{610} \\
& +z_{110} z_{29} z_{610}+2 z_{29} z_{37} z_{610}-2 z_{19} z_{39} z_{610}+2 z_{25} z_{410} z_{610}-z_{39} z_{45} z_{610} \\
& -z_{29} z_{46} z_{610}-z_{28} z_{47} z_{610}+z_{19} z_{48} z_{610}+2 z_{45} z_{48} z_{610}+z_{18} z_{49} z_{610} \\
& -z_{35} z_{49} z_{610}+z_{24} z_{510} z_{610}-3 z_{34} z_{59} z_{610}+z_{210}^{2} z_{67}-3 z_{29} z_{310} z_{67} \\
& +2 z_{39}^{2} z_{67}+3 z_{28} z_{410} z_{67}-2 z_{39} z_{48} z_{67}+2 z_{48}^{2} z_{67}-2 z_{38} z_{49} z_{67} \\
& -z_{19} z_{410} z_{68}+z_{27} z_{410} z_{68}-z_{39} z_{47} z_{68}+z_{47} z_{48} z_{68}+z_{37} z_{49} z_{68} \\
& -z_{46} z_{49} z_{68}-z_{110} z_{210} z_{69}+2 z_{19} z_{310} z_{69}+z_{27} z_{310} z_{69}-2 z_{37} z_{39} z_{69} \\
& -z_{18} z_{410} z_{69}-2 z_{26} z_{410} z_{69}+2 z_{39} z_{46} z_{69}+z_{38} z_{47} z_{69}-2 z_{46} z_{48} z_{69} \\
& +z_{36} z_{49} z_{69}-z_{24} z_{610} z_{69}+2 z_{34} z_{69}^{2}+z_{210} z_{26} z_{710}-z_{110} z_{28} z_{710} \\
& -2 z_{25} z_{310} z_{710}-z_{29} z_{36} z_{710}-z_{28} z_{37} z_{710}+z_{19} z_{38} z_{710}+z_{18} z_{39} z_{710}
\end{aligned}
$$




$$
\begin{aligned}
& +2 z_{35} z_{39} z_{710}-z_{38} z_{45} z_{710}+2 z_{28} z_{46} z_{710}-2 z_{18} z_{48} z_{710}-z_{35} z_{48} z_{710} \\
& -z_{23} z_{510} z_{710}+3 z_{34} z_{58} z_{710}-z_{24} z_{68} z_{710}+2 z_{23} z_{69} z_{71}+z_{110} z_{210} z_{78} \\
& -z_{19} z_{310} z_{78}-2 z_{27} z_{310} z_{78}+2 z_{37} z_{39} z_{78}+2 z_{18} z_{410} z_{78}+z_{26} z_{410} z_{78} \\
& -z_{38} z_{47} z_{78}-2 z_{37} z_{48} z_{78}+2 z_{46} z_{48} z_{78}-z_{36} z_{49} z_{78}+2 z_{24} z_{610} z_{78} \\
& -2 z_{34} z_{69} z_{78}-z_{23} z_{710} z_{78}+2 z_{34} z_{78}^{2}-z_{18} z_{310} z_{79}+z_{26} z_{310} z_{79} \\
& +z_{37} z_{38} z_{79}-z_{36} z_{39} z_{79}-z_{38} z_{46} z_{79}+z_{36} z_{48} z_{79}-z_{23} z_{610} z_{79} \\
& -2 z_{34} z_{68} z_{79}-z_{110} z_{19} z_{810}+z_{17} z_{210} z_{810}-2 z_{27} z_{37} z_{810}+2 z_{17} z_{39} z_{810} \\
& -2 z_{15} z_{410} z_{810}+z_{37} z_{45} z_{810}+z_{27} z_{46} z_{810}-2 z_{45} z_{46} z_{810}+z_{26} z_{47} z_{810} \\
& +z_{35} z_{47} z_{810}-z_{17} z_{48} z_{810}-z_{16} z_{49} z_{810}-z_{14} z_{510} z_{810}+3 z_{34} z_{57} z_{810} \\
& +3 z_{24} z_{67} z_{810}-2 z_{14} z_{69} z_{810}+2 z_{12} z_{710} z_{810}+z_{14} z_{78} z_{810}+z_{13} z_{79} z_{810} \\
& +z_{110}^{2} z_{89}-3 z_{17} z_{310} z_{89}+2 z_{37}^{2} z_{89}+3 z_{16} z_{410} z_{89}-2 z_{37} z_{46} z_{89} \\
& +2 z_{46}^{2} z_{89}-2 z_{36} z_{47} z_{89}+3 z_{14} z_{610} z_{89}-6 z_{34} z_{67} z_{89}-3 z_{13} z_{710} z_{89} \\
& +z_{110} z_{18} z_{910}-z_{16} z_{210} z_{910}+2 z_{15} z_{310} z_{910}+z_{27} z_{36} z_{910}+z_{26} z_{37} z_{910} \\
& -2 z_{35} z_{37} z_{910}-z_{17} z_{38} z_{910}-z_{16} z_{39} z_{910}+z_{36} z_{45} z_{910}-2 z_{26} z_{46} z_{910} \\
& +z_{35} z_{46} z_{910}+2 z_{16} z_{48} z_{910}+z_{13} z_{510} z_{910}-3 z_{34} z_{56} z_{910}-2 z_{12} z_{610} z_{910} \\
& -3 z_{23} z_{67} z_{910}+z_{14} z_{68} z_{910}+z_{13} z_{69} z_{910}-2 z_{13} z_{78} z_{910} \text {. }
\end{aligned}
$$

Lemma 3.2. For every $A \in G L_{5}, B \in G L_{8}, \tilde{Z} \in \mathrm{Alt}_{10}$, we have

$$
\tilde{Z} \mapsto(\operatorname{det} A)^{4}(\operatorname{det} B){ }^{t} \Lambda_{2}(A)^{-1} \tilde{Z} \Lambda_{2}(A)^{-1}
$$

and

(3.4) $\Psi\left((\operatorname{det} A)^{4}(\operatorname{det} B)^{t} \Lambda_{2}(A)^{-1} \tilde{Z} \Lambda_{2}(A)^{-1}\right)=(\operatorname{det} A)^{10}(\operatorname{det} B)^{3}{ }^{t} A^{-1} \Psi(\tilde{Z}) A^{-1}$.

Proof. It is enough to prove the equivariance (3.4) in the case when $A$ is one of the fundamental matrices,

$$
A_{u}=\left(\begin{array}{ccccc}
1 & \varepsilon & & & \\
& 1 & & & \\
& & 1 & & \\
& & & 1 & \\
& & & & 1
\end{array}\right), A_{d}=\operatorname{diag}(a, 1,1,1,1),
$$

or permutation matrices.

For the diagonal or permutation matrices, verifying (3.4) is easy. Note that, for $A_{d}=\operatorname{diag}\left(a_{1}, a_{2}, a_{3}, a_{4}, a_{5}\right)$, we have

$$
\begin{gathered}
\operatorname{det} A_{d}=a_{1} a_{2} a_{3} a_{4} a_{5} \text { and } z_{12} \mapsto\left(\operatorname{det} A_{d}\right)^{3} a_{1}^{-1} a_{4} a_{5}, \\
z_{13} \mapsto\left(\operatorname{det} A_{d}\right)^{3} a^{-1} a_{3} a_{5}, \cdots, z_{910} \mapsto\left(\operatorname{det} A_{d}\right)^{3} a_{1} a_{2} a_{5}^{-1} .
\end{gathered}
$$

Hence, we have $\psi_{i j} \mapsto\left(\operatorname{det} A_{d}\right)^{10} a_{i}^{-1} a_{j}^{-1},(1 \leq i<j \leq 5)$ and then

$$
\Psi\left(\left(\operatorname{det} A_{d}\right)^{t} \Lambda_{2}\left(A_{d}\right) \tilde{Z} \Lambda_{2}\left(A_{d}\right)^{-1}\right)=\left(\operatorname{det} A_{d}\right)^{10 t} A_{d}^{-1} \Psi(\tilde{Z}) A_{d}^{-1} .
$$

For $A_{u}$, we consider the action of $A_{u}$. Since $\operatorname{det} A_{u}=1$, and

$$
\Psi(\tilde{Z}) \mapsto \Psi\left({ }^{t} \Lambda_{2}\left(A_{u}\right) \tilde{Z} \Lambda_{2}\left(A_{u}\right)^{-1}\right),
$$

then we have $\psi_{2 j} \mapsto \psi_{2 j}-\varepsilon \psi_{1 j}(3 \leq j \leq 5), \psi_{1 j} \mapsto \psi_{1 j}$ and $\psi_{l k} \mapsto \psi_{l k}$ $(3 \leq l<k \leq 5)$. Hence, we have $\Psi\left({ }^{t} \Lambda_{2}\left(A_{u}\right)^{-1} \tilde{Z} \Lambda_{2}\left(A_{u}\right)^{-1}\right)={ }^{t} A_{u}^{-1} \Psi(\tilde{Z}) A_{u}^{-1}$. 
Remark B. We can construct the above polynomials $\psi_{i j}(1 \leq i<j \leq 5)$ in the same program as in Remark A. This case is much more complicated than the case of $\varphi_{i j}^{\prime} s$. Here we note that we consider constructing the polynomial $\psi_{45}$. First, we consider the polynomial corresponding to the weight $(\operatorname{det} A)^{10} a_{4}^{-1} a_{5}^{-1}$. This polynomial is constructed of 205-term monomials. After the action of the generators of $G L_{5}$, we have uniquely the polynomial $\psi_{45}$ constructed of 148-term monomials. Hence from the explicit form of $\psi_{45}$, we can construct the other polynomials $\psi_{i j}$ by the action of $G L_{5}$.

Step 5. From (3.2) and (3.3), $\Phi(\tilde{Z}) \Delta(X \cdot Y) \mapsto(\operatorname{det} A)^{8}(\operatorname{det} B)^{2} A \Phi(\tilde{Z}) \Delta(X$. $Y) A^{-1}$ and hence $F_{1}(x)=\operatorname{tr} \Phi(\tilde{Z}) \Delta(X \cdot Y)$ is a relative invariant of degree 24 corresponding to the character $(\operatorname{det} A)^{8}(\operatorname{det} B)^{2}$.

From $(3.1)$ and $(3.4),(X \cdot Y) \Psi(\tilde{Z}) \mapsto(\operatorname{det} A)^{10}(\operatorname{det} B)^{3} A(X \cdot Y) \Psi(\tilde{Z}) A^{-1}$ and hence $F_{2}(x)=\operatorname{tr}((X \cdot Y) \Psi(\tilde{Z}))$ is a relative invariant of degree 26 corresponding to the character $(\operatorname{det} A)^{10}(\operatorname{det} B)^{3}$.

For a generic point $x_{0}=\left(e_{1} \wedge e_{2}, 2 e_{1} \wedge e_{3}, 2 e_{2} \wedge e_{3}, e_{1} \wedge e_{5}, e_{1} \wedge e_{4}-e_{2} \wedge\right.$ $\left.e_{5}, e_{2} \wedge e_{4}-e_{3} \wedge e_{5}, e_{3} \wedge e_{4}, e_{4} \wedge e_{5}, e_{2}^{\prime}+e_{8}^{\prime}\right)$, we have

$$
\begin{aligned}
& X_{0} \cdot Y_{0}=\left(\begin{array}{ccccc}
0 & & 2 & & \\
& 0 & & & \\
-2 & & 0 & & \\
& & & 0 & 1 \\
& & & -1 & 0
\end{array}\right) \text { and } \Delta\left(X_{0} \cdot Y_{0}\right)=\left(\begin{array}{ccccc}
0 & & & & \\
& 4 & & & \\
& & 0 & & \\
& & 0 & \\
& & & 0
\end{array}\right) \text {, }
\end{aligned}
$$

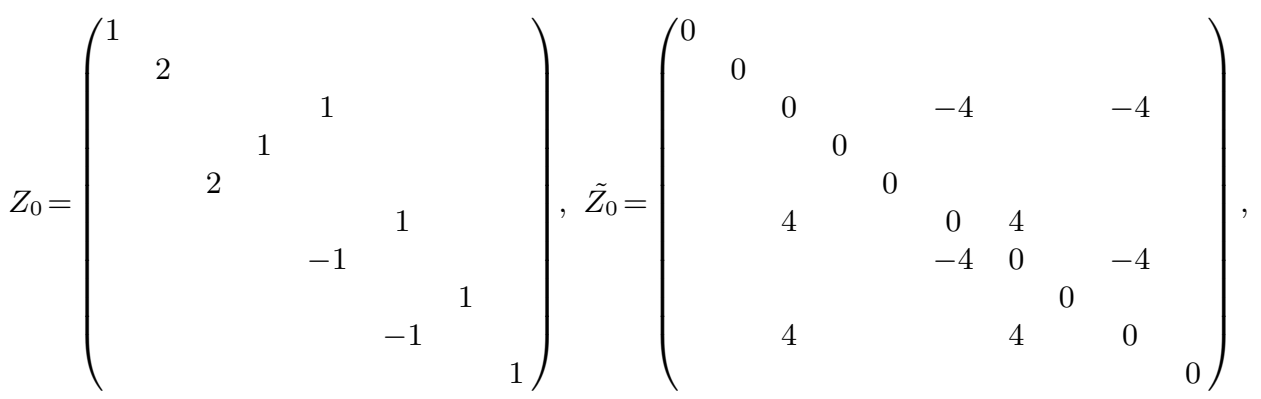

$$
\begin{aligned}
& \Phi\left(\tilde{Z}_{0}\right)=\left(\begin{array}{ccccc}
0 & & & & \\
& 16 & & & \\
& & 0 & & \\
& & & 0 & \\
& & & & 0
\end{array}\right), \Psi\left(\tilde{Z}_{0}\right)=\left(\begin{array}{ccccc}
0 & & & & \\
& 0 & & & \\
& & 0 & & \\
& & & 0 & 192 \\
& & & -192 & 0
\end{array}\right),
\end{aligned}
$$

and hence

$$
\begin{aligned}
& F_{1}\left(X_{1}^{0}, \cdots, X_{8}^{0}, Y_{0}\right)=\operatorname{tr}\left(\Phi\left(\tilde{Z}_{0}\right) \Delta\left(X_{0} \cdot Y_{0}\right)\right) \\
& =\operatorname{tr}\left(\left(\begin{array}{ccccc}
0 & & & & \\
& 16 & & & \\
& & 0 & & \\
& & & 0 & \\
& & & & 0
\end{array}\right)\left(\begin{array}{ccccc}
0 & & & & \\
& 4 & & & \\
& & 0 & & \\
& & & 0 & \\
& & & & 0
\end{array}\right)\right)=64 \neq 0,
\end{aligned}
$$




$$
\begin{aligned}
F_{2}\left(X_{1}^{0}, \cdots, X_{8}^{0}, Y_{0}\right)=\operatorname{tr}\left(\left(X_{0} \cdot Y_{0}\right) \Psi\left(\tilde{Z}_{0}\right)\right) \\
=\operatorname{tr}\left(\left(\begin{array}{ccccc}
0 & & 2 & & \\
& 0 & & & \\
-2 & & 0 & & \\
& & & 0 & 1 \\
& & & -1 & 0
\end{array}\right)\left(\begin{array}{ccccc}
0 & & & & \\
& 0 & & & \\
& & 0 & & \\
& & & 0 & 192 \\
& & & -192 & 0
\end{array}\right)\right)=-384 \neq 0 .
\end{aligned}
$$

Thus they are not identically zero.

Since $\operatorname{deg} F_{1}=24<\operatorname{deg} F_{2}=26$ and $\operatorname{deg}_{Y} F_{1}=4>\operatorname{deg}_{Y} F_{2}=1$, we have $F_{1} \nmid F_{2}$ and $F_{2} \nmid F_{1}$. Hence, if $F_{1}$ or $F_{2}$ is not irreducible, it is a power of an irreducible invariant. Since the character corresponding to $F_{2}$ is $(\operatorname{det} A)^{10}(\operatorname{det} B)^{3}$, we have the irreducibility of $F_{2}$. If $F_{1}$ is not irreducible, we have $F_{1}=G^{2}$ for some relative invariant corresponding to the character $(\operatorname{det} A)^{4}(\operatorname{det} B)$. For

$$
\begin{aligned}
\tilde{x}=\left(\left(z_{1}^{(1)} e_{1} \wedge e_{2}, 2 z_{2}^{(2)} e_{1} \wedge e_{3}, 2 z_{5}^{(3)} e_{2} \wedge e_{3}, z_{4}^{(4)} e_{1} \wedge e_{5}, z_{3}^{(5)} e_{1} \wedge e_{4}\right.\right. \\
\left.\left.\quad-z_{7}^{(5)} e_{2} \wedge e_{5}, z_{6}^{(6)} e_{2} \wedge e_{4}-z_{9}^{(6)} e_{3} \wedge e_{5}, z_{8}^{(7)} e_{3} \wedge e_{4}, z_{10}^{(8)} e_{4} \wedge e_{5}\right), e_{2}^{\prime}+e_{8}^{\prime}\right) \in V,
\end{aligned}
$$

we have

$$
F_{1}(\tilde{x})=64\left(z_{1}^{(1)} z_{2}^{(2)} z_{5}^{(3)} z_{4}^{(4)} z_{5}^{(7)} z_{6}^{(6)} z_{8}^{(7)} z_{10}^{(8)}\right)^{2} z_{2}^{(2)} z_{10}^{(8)}
$$

and hence $F_{1}(x)$ is not of the form $G(x)^{2}$ for some polynomial $G(x)$. This shows the irreducibility of $F_{1}(x)$.

Theorem 3.3. The prehomogeneous vector space $\left(G L_{5} \times G L_{8}, \Lambda_{2} \otimes \Lambda_{1}+1 \otimes \Lambda_{1}^{*}\right.$, $\left.\mathrm{Alt}_{5}^{\oplus 8} \oplus V(8)^{*}\right)$ has 2 basic relative invariants:

(1) $F_{1}(x)=\operatorname{tr} \Phi(\tilde{Z}) \Delta(X \cdot Y) \longleftrightarrow(\operatorname{det} A)^{8}(\operatorname{det} B)^{2}, \operatorname{deg} \cdot F_{1}=24$,

(2) $F_{2}(x)=\operatorname{tr}(X \cdot Y) \Psi(\tilde{Z}) \longleftrightarrow(\operatorname{det} A)^{10}(\operatorname{det} B)^{3}, \operatorname{deg} \cdot F_{2}=26$.

3.2. Explicit construction of the irreducible relative invariants of $\left(G L_{1}^{2} \times\right.$ $\left.\operatorname{Spin}_{10} \times S L_{14}, \Lambda^{\prime} \otimes \Lambda_{1}+1 \otimes \Lambda_{1}^{*}\right)$ with $H \sim S L_{2} \times S O_{2}, N=2$. We may assume that $G L_{1} \times \operatorname{Spin}_{10} \times G L_{14}$ acts on $V=V(16)^{\oplus 14} \oplus M(14,1)$ by $x=\left(\left(X_{1}, X_{2}, \cdots, X_{14}\right), Y\right)$ $\mapsto\left(\left(\Lambda^{\prime}(A) X_{1}, \Lambda^{\prime}(A) X_{2}, \cdots, \Lambda^{\prime}(A) X_{14}\right)^{t} B, \alpha^{t} B^{-1} Y\right)$ for $x=\left(\left(X_{1}, X_{2}, \cdots, X_{14}\right), Y\right)$ $\in V$ and $g=(\alpha, A, B) \in G$.

For $Y={ }^{t}\left(y_{1}, \cdots, y_{14}\right), X \cdot Y:=X_{1} y_{1}+\cdots+X_{14} y_{14} \in V(16)$. Then we have $X \cdot Y \longmapsto \alpha \Lambda^{\prime}(A) X \cdot Y$. Now we define the mapping

$$
\eta: V(16) \longrightarrow V(10), X=\left(x_{0}, x_{12}, \cdots, x_{2345}\right) \longmapsto \eta(X)=\left(\eta_{1}(x), \ldots, \eta_{10}(x)\right)
$$

as follows:

$$
\begin{aligned}
& \eta_{1}(x)=-x_{12} x_{1345}+x_{13} x_{1245}-x_{14} x_{1235}+x_{15} x_{1234}, \\
& \eta_{2}(x)=x_{23} x_{1245}-x_{24} x_{1235}+x_{25} x_{1234}-x_{12} x_{2345} \text {, } \\
& \eta_{3}(x)=-x_{34} x_{1235}+x_{35} x_{1234}-x_{13} x_{2345}+x_{23} x_{1345} \\
& \eta_{4}(x)=x_{45} x_{1234}-x_{14} x_{2345}+x_{24} x_{1345}-x_{34} x_{1245}, \\
& \eta_{5}(x)=-x_{15} x_{2345}+x_{25} x_{1345}-x_{35} x_{1245}+x_{45} x_{1235}, \\
& \eta_{6}(x)=\begin{array}{llll}
x_{0} x_{2345} & -x_{23} x_{45} & +x_{24} x_{35} & -x_{25} x_{34},
\end{array} \\
& \eta_{7}(x)=-x_{0} x_{1345}+x_{34} x_{15} \quad-x_{35} x_{14} \quad+x_{13} x_{45}, \\
& \eta_{8}(x)=x_{0} x_{1245} \quad-x_{45} x_{12} \quad+x_{14} x_{25} \quad-x_{24} x_{15}, \\
& \eta_{9}(x)=-x_{0} x_{1235}+x_{15} x_{23} \quad-x_{25} x_{13} \quad+x_{35} x_{12}, \\
& \eta_{10}(x)=\begin{array}{llll}
x_{0} x_{1234} & -x_{12} x_{34} & +x_{13} x_{24} & -x_{14} x_{23}
\end{array}
\end{aligned}
$$


(cf. [7], [8]). Then we have

$$
\eta\left(\alpha \Lambda^{\prime}(A)(X \cdot Y)\right)=\alpha^{2} \chi(A) \eta(X \cdot Y),
$$

where $\chi$ is the vector representation of $\operatorname{Spin}_{10}$. Since the infinitesimal representation of $\chi\left(\right.$ resp. $\left.\Lambda^{\prime}\right)$ is given by (5.28) (resp. (5.38)) in [1, we have

$$
\chi\left(\operatorname{Spin}_{10}\right)=S O(10, K)=\left\{A \in S L_{10} ;{ }^{t} A K A=K\right\} \text { for } K=\left(\begin{array}{c|c}
O & I_{5} \\
\hline I_{5} & O
\end{array}\right) .
$$

On the other hand, we put

$$
\begin{aligned}
\tilde{X}_{i}= & { }^{t}\left(x_{0}^{(i)}, x_{12}^{(i)}, x_{13}^{(i)}, x_{14}^{(i)}, x_{15}^{(i)}, x_{23}^{(i)}, x_{24}^{(i)}, x_{25}^{(i)},\right. \\
& \left.\quad x_{34}^{(i)}, x_{35}^{(i)}, x_{45}^{(i)}, x_{1234}^{(i)}, x_{1235}^{(i)}, x_{1245}^{(i)}, x_{1345}^{(i)}, x_{2345}^{(i)}\right) \\
& \in V(16)
\end{aligned}
$$

and consider that $Z=\left[\tilde{X}_{1}, \cdots, \tilde{X_{14}}\right] \in M(16,14)$.

Let $Z^{(i, j)}$ be the $14 \times 14$-matrix obtained from $\tilde{Z}$ by substracting the $i$-th and the $j$-th rows and $\tilde{Z}:=\left(z_{i j}\right) \in \operatorname{Alt}_{16}$ with $z_{i j}:=(-1)^{i+j} \operatorname{det} Z^{(i, j)}$. Then, by Lemma 2.3 , we have

$$
\tilde{Z} \longmapsto\left(\operatorname{det} \Lambda^{\prime}(A)\right)(\operatorname{det} B){ }^{t} \Lambda^{\prime}(A)^{-1} \tilde{Z} \Lambda^{\prime}(A)^{-1} .
$$

Note that $\operatorname{det} \Lambda^{\prime}(A)=1$. Now we shall construct $\Phi(\tilde{Z}) \in \operatorname{Sym}_{10}$ such that

$$
\Phi(\tilde{Z}) \longmapsto(\operatorname{det} B)^{2}{ }^{t} \chi(A)^{-1} \Phi(\tilde{Z}) \chi(A)^{-1} .
$$

If we put

$$
\begin{aligned}
\Phi(\tilde{Z}) & =\left(\varphi(\tilde{Z})_{i, j}\right)_{1 \leq i<j \leq 10} \\
& =\Phi(\tilde{Z})=\left(\begin{array}{ccc|ccc}
\varphi_{11} & \cdots & \varphi_{15} & \varphi_{16} & \cdots & \varphi_{110} \\
\vdots & \ddots & \vdots & \vdots & \ddots & \vdots \\
\varphi_{15} & \cdots & \varphi_{55} & \varphi_{56} & \cdots & \varphi_{510} \\
\hline \varphi_{16} & \cdots & \varphi_{56} & \varphi_{66} & \cdots & \varphi_{610} \\
\vdots & \ddots & \vdots & \vdots & \ddots & \vdots \\
\varphi_{110} & \cdots & \varphi_{510} & \varphi_{610} & \cdots & \varphi_{1010}
\end{array}\right) \in \operatorname{Sym}_{10}
\end{aligned}
$$

with the following entries, then we have Lemma 3.4.

$$
\begin{aligned}
& \varphi_{11}:=-z_{215}^{2}+2 z_{1415} z_{23}-2 z_{1315} z_{24}+2 z_{1215} z_{25}-z_{314}^{2}+2 z_{214} z_{315}+2 z_{1314} z_{34}- \\
& 2 z_{1214} z_{35}-z_{413}^{2}+2 z_{313} z_{414}-2 z_{213} z_{415}+2 z_{1213} z_{45}-z_{512}^{2}+2 z_{412} z_{513}-2 z_{312} z_{514}+ \\
& 2 z_{212} z_{515}, \\
& \varphi_{22}:=-z_{216}^{2}+2 z_{1416} z_{26}-2 z_{1316} z_{27}+2 z_{1216} z_{28}-z_{614}^{2}+2 z_{214} z_{616}+2 z_{1314} z_{67}- \\
& 2 z_{1214} z_{68}-z_{713}^{2}+2 z_{613} z_{714}-2 z_{213} z_{716}+2 z_{1213} z_{78}-z_{812}^{2}+2 z_{712} z_{813}-2 z_{612} z_{814}+ \\
& 2 z_{212} z_{816}, \\
& \varphi_{33}:=-z_{1012}^{2}+2 z_{1216} z_{310}+2 z_{1016} z_{312}-z_{316}^{2}+2 z_{1516} z_{36}-2 z_{1316} z_{39}-2 z_{1215} z_{610}- \\
& 2 z_{1015} z_{612}-z_{615}^{2}+2 z_{315} z_{616}+2 z_{1315} z_{69}+2 z_{1213} z_{910}+2 z_{1013} z_{912}-z_{913}^{2}+2 z_{613} z_{915}- \\
& 2 z_{313} z_{916}, \\
& \varphi_{44}:=-z_{1112}^{2}+2 z_{1216} z_{411}+2 z_{1116} z_{412}-z_{416}^{2}+2 z_{1516} z_{47}-2 z_{1416} z_{49}-2 z_{1215} z_{711}- \\
& 2 z_{1115} z_{712}-z_{715}^{2}+2 z_{415} z_{716}+2 z_{1415} z_{79}+2 z_{1214} z_{911}+2 z_{1114} z_{912}-z_{914}^{2}+2 z_{714} z_{915}- \\
& 2 z_{414} z_{916}, \\
& \varphi_{55}:=-z_{1014}^{2}-z_{1113}^{2}+2 z_{1013} z_{1114}+2 z_{1011} z_{1314}-2 z_{1416} z_{510}+2 z_{1316} z_{511}+2 z_{1116} z_{513}- \\
& 2 z_{1016} z_{514}-z_{516}^{2}+2 z_{1516} z_{58}+2 z_{1415} z_{810}-2 z_{1315} z_{811}-2 z_{1115} z_{813}+2 z_{1015} z_{814}- \\
& z_{815}^{2}+2 z_{515} z_{816},
\end{aligned}
$$


$\varphi_{66}:=-z_{116}^{2}+2 z_{1116} z_{16}-2 z_{1016} z_{17}-z_{611}^{2}+2 z_{111} z_{616}+2 z_{1011} z_{67}-z_{710}^{2}+2 z_{610} z_{711}-$ $2 z_{110} z_{716}+2 z_{79} z_{810}-2 z_{69} z_{811}+2 z_{19} z_{816}-z_{89}^{2}+2 z_{78} z_{910}-2 z_{68} z_{911}+2 z_{18} z_{916}$, $\varphi_{77}:=-z_{115}^{2}+2 z_{1115} z_{13}-2 z_{1015} z_{14}-z_{311}^{2}+2 z_{111} z_{315}+2 z_{1011} z_{34}-z_{410}^{2}+2 z_{310} z_{411}-$ $2 z_{110} z_{415}+2 z_{49} z_{510}-2 z_{39} z_{511}+2 z_{19} z_{515}-z_{59}^{2}+2 z_{45} z_{910}-2 z_{35} z_{911}+2 z_{15} z_{915}$, $\varphi_{88}:=-z_{114}^{2}+2 z_{1114} z_{12}-z_{211}^{2}+2 z_{111} z_{214}+2 z_{28} z_{411}-2 z_{18} z_{414}-z_{48}^{2}-2 z_{27} z_{511}+$ $2 z_{17} z_{514}-z_{57}^{2}+2 z_{47} z_{58}-2 z_{25} z_{711}+2 z_{15} z_{714}+2 z_{45} z_{78}+2 z_{24} z_{811}-2 z_{14} z_{814}$ $\varphi_{99}:=-z_{113}^{2}+2 z_{1013} z_{12}-z_{210}^{2}+2 z_{110} z_{213}+2 z_{28} z_{310}-2 z_{18} z_{313}-z_{38}^{2}-2 z_{26} z_{510}+$ $2 z_{16} z_{513}-z_{56}^{2}+2 z_{36} z_{58}-2 z_{25} z_{610}+2 z_{15} z_{613}+2 z_{35} z_{68}+2 z_{23} z_{810}-2 z_{13} z_{813}$, $\varphi_{1010}:=-z_{112}^{2}+2 z_{19} z_{212}-z_{29}^{2}-2 z_{17} z_{312}-z_{37}^{2}+2 z_{27} z_{39}+2 z_{16} z_{412}-z_{46}^{2}+2 z_{36} z_{47}-$ $2 z_{26} z_{49}+2 z_{14} z_{612}+2 z_{34} z_{67}-2 z_{24} z_{69}-2 z_{13} z_{712}+2 z_{23} z_{79}+2 z_{12} z_{912}$,

$\varphi_{12}:=-z_{215} z_{216}+z_{1416} z_{23}-z_{1316} z_{24}+z_{1216} z_{25}+z_{1415} z_{26}-z_{1315} z_{27}+z_{1215} z_{28}+$ $z_{214} z_{316}+z_{1314} z_{37}-z_{1214} z_{38}-z_{213} z_{416}-z_{1314} z_{46}+z_{1213} z_{48}+z_{212} z_{516}+z_{1214} z_{56}-$ $z_{1213} z_{57}-z_{514} z_{612}+z_{414} z_{613}-z_{314} z_{614}+z_{214} z_{615}+z_{513} z_{712}-z_{413} z_{713}+z_{313} z_{714}-$ $z_{213} z_{715}-z_{512} z_{812}+z_{412} z_{813}-z_{312} z_{814}+z_{212} z_{815}$,

$\varphi_{13}:=z_{1215} z_{210}+z_{1015} z_{212}+z_{1516} z_{23}-z_{1315} z_{29}-z_{1214} z_{310}-z_{1014} z_{312}-z_{216} z_{315}+$ $z_{314} z_{316}-z_{1316} z_{34}+z_{1216} z_{35}+z_{1415} z_{36}+z_{1314} z_{39}+z_{1213} z_{410}+z_{1013} z_{412}-z_{313} z_{416}-$ $z_{1315} z_{46}-z_{1012} z_{512}+z_{312} z_{516}+z_{1215} z_{56}-z_{1213} z_{59}-z_{515} z_{612}+z_{415} z_{613}-z_{315} z_{614}+$ $z_{215} z_{615}+z_{513} z_{912}-z_{413} z_{913}+z_{313} z_{914}-z_{213} z_{915}$,

$\varphi_{14}:=z_{1215} z_{211}+z_{1115} z_{212}+z_{1516} z_{24}-z_{1415} z_{29}-z_{1214} z_{311}-z_{1114} z_{312}-z_{1416} z_{34}+$ $z_{1415} z_{37}+z_{1213} z_{411}+z_{1113} z_{412}+z_{316} z_{414}-z_{216} z_{415}-z_{413} z_{416}+z_{1216} z_{45}-z_{1315} z_{47}+$ $z_{1314} z_{49}-z_{1112} z_{512}+z_{412} z_{516}+z_{1215} z_{57}-z_{1214} z_{59}-z_{515} z_{712}+z_{415} z_{713}-z_{315} z_{714}+$ $z_{215} z_{715}+z_{514} z_{912}-z_{414} z_{913}+z_{314} z_{914}-z_{214} z_{915}$

$\varphi_{15}:=-z_{1415} z_{210}+z_{1315} z_{211}+z_{1115} z_{213}-z_{1015} z_{214}+z_{1516} z_{25}-z_{1314} z_{311}-z_{1114} z_{313}+$ $z_{1014} z_{314}-z_{1416} z_{35}+z_{1415} z_{38}+z_{1314} z_{410}+z_{1113} z_{413}-z_{1013} z_{414}+z_{1316} z_{45}-z_{1315} z_{48}-$ $z_{1214} z_{510}+z_{1213} z_{511}-z_{1112} z_{513}-z_{416} z_{513}+z_{1012} z_{514}+z_{316} z_{514}-z_{216} z_{515}+z_{512} z_{516}+$ $z_{1215} z_{58}-z_{515} z_{812}+z_{415} z_{813}-z_{315} z_{814}+z_{215} z_{815}$,

$\varphi_{16}:=z_{1316} z_{14}-z_{1216} z_{15}-z_{1415} z_{16}+z_{115} z_{216}-z_{1116} z_{23}+z_{1015} z_{27}-z_{114} z_{316}-$ $z_{1014} z_{37}+z_{113} z_{416}-z_{1113} z_{46}-z_{112} z_{516}+z_{1112} z_{56}-z_{1012} z_{57}+z_{511} z_{612}-z_{411} z_{613}+$ $z_{311} z_{614}-z_{211} z_{615}+z_{410} z_{713}-z_{310} z_{714}+z_{210} z_{715}-z_{513} z_{79}-z_{412} z_{810}+z_{59} z_{812}+$ $z_{39} z_{814}-z_{29} z_{815}-z_{48} z_{913}+z_{38} z_{914}-z_{28} z_{915}$

$\varphi_{17}:=-z_{1315} z_{14}+z_{13} z_{1415}+z_{1215} z_{15}-z_{115} z_{215}+z_{1115} z_{23}-z_{1015} z_{24}-z_{311} z_{314}+$ $z_{114} z_{315}+z_{211} z_{315}+z_{1014} z_{34}-z_{1113} z_{34}+z_{1112} z_{35}+z_{313} z_{411}-z_{410} z_{413}+z_{310} z_{414}-$ $z_{113} z_{415}-z_{210} z_{415}-z_{1012} z_{45}+z_{412} z_{510}-z_{312} z_{511}+z_{49} z_{513}-z_{39} z_{514}+z_{112} z_{515}+$ $z_{29} z_{515}-z_{512} z_{59}+z_{45} z_{913}-z_{35} z_{914}+z_{25} z_{915}$

$\varphi_{18}:=z_{1314} z_{14}-z_{12} z_{1415}-z_{1214} z_{15}+z_{115} z_{214}-z_{211} z_{215}-z_{1114} z_{23}+z_{1113} z_{24}-$ $z_{1112} z_{25}+z_{214} z_{311}-z_{114} z_{314}-z_{213} z_{411}+z_{113} z_{414}-z_{38} z_{414}+z_{28} z_{415}+z_{413} z_{48}+$ $z_{212} z_{511}-z_{47} z_{513}-z_{112} z_{514}+z_{37} z_{514}-z_{27} z_{515}+z_{512} z_{57}-z_{412} z_{58}-z_{45} z_{713}+z_{35} z_{714}-$ $z_{25} z_{715}+z_{45} z_{812}-z_{34} z_{814}+z_{24} z_{815}$

$\varphi_{19}:=-z_{13} z_{1314}+z_{12} z_{1315}+z_{1213} z_{15}-z_{115} z_{213}+z_{210} z_{215}+z_{1014} z_{23}-z_{1013} z_{24}+$ $z_{1012} z_{25}-z_{214} z_{310}+z_{114} z_{313}-z_{28} z_{315}+z_{314} z_{38}+z_{213} z_{410}-z_{113} z_{413}-z_{313} z_{48}-$ $z_{212} z_{510}+z_{112} z_{513}+z_{46} z_{513}-z_{36} z_{514}+z_{26} z_{515}-z_{512} z_{56}+z_{312} z_{58}+z_{45} z_{613}-z_{35} z_{614}+$ $z_{25} z_{615}-z_{35} z_{812}+z_{34} z_{813}-z_{23} z_{815}$,

$\varphi_{110}:=-z_{12} z_{1215}+z_{1214} z_{13}-z_{1213} z_{14}+z_{115} z_{212}-z_{215} z_{29}-z_{114} z_{312}+z_{27} z_{315}-$ $z_{314} z_{37}+z_{214} z_{39}+z_{113} z_{412}+z_{36} z_{414}-z_{26} z_{415}-z_{413} z_{46}+z_{313} z_{47}-z_{213} z_{49}-z_{112} z_{512}+$ $z_{412} z_{56}-z_{312} z_{57}+z_{212} z_{59}-z_{45} z_{612}+z_{34} z_{614}-z_{24} z_{615}+z_{35} z_{712}-z_{34} z_{713}+z_{23} z_{715}-$ $z_{25} z_{912}+z_{24} z_{913}-z_{23} z_{914}$

$\varphi_{23}:=z_{1216} z_{210}+z_{1016} z_{212}+z_{1516} z_{26}-z_{1316} z_{29}-z_{216} z_{316}+z_{1416} z_{36}-z_{1316} z_{37}+$ $z_{1216} z_{38}-z_{1214} z_{610}-z_{1014} z_{612}-z_{614} z_{615}+z_{215} z_{616}+z_{314} z_{616}+z_{1315} z_{67}-z_{1215} z_{68}+$ $z_{1314} z_{69}+z_{1213} z_{710}+z_{1013} z_{712}+z_{613} z_{715}-z_{313} z_{716}-z_{1012} z_{812}-z_{612} z_{815}+z_{312} z_{816}-$ 


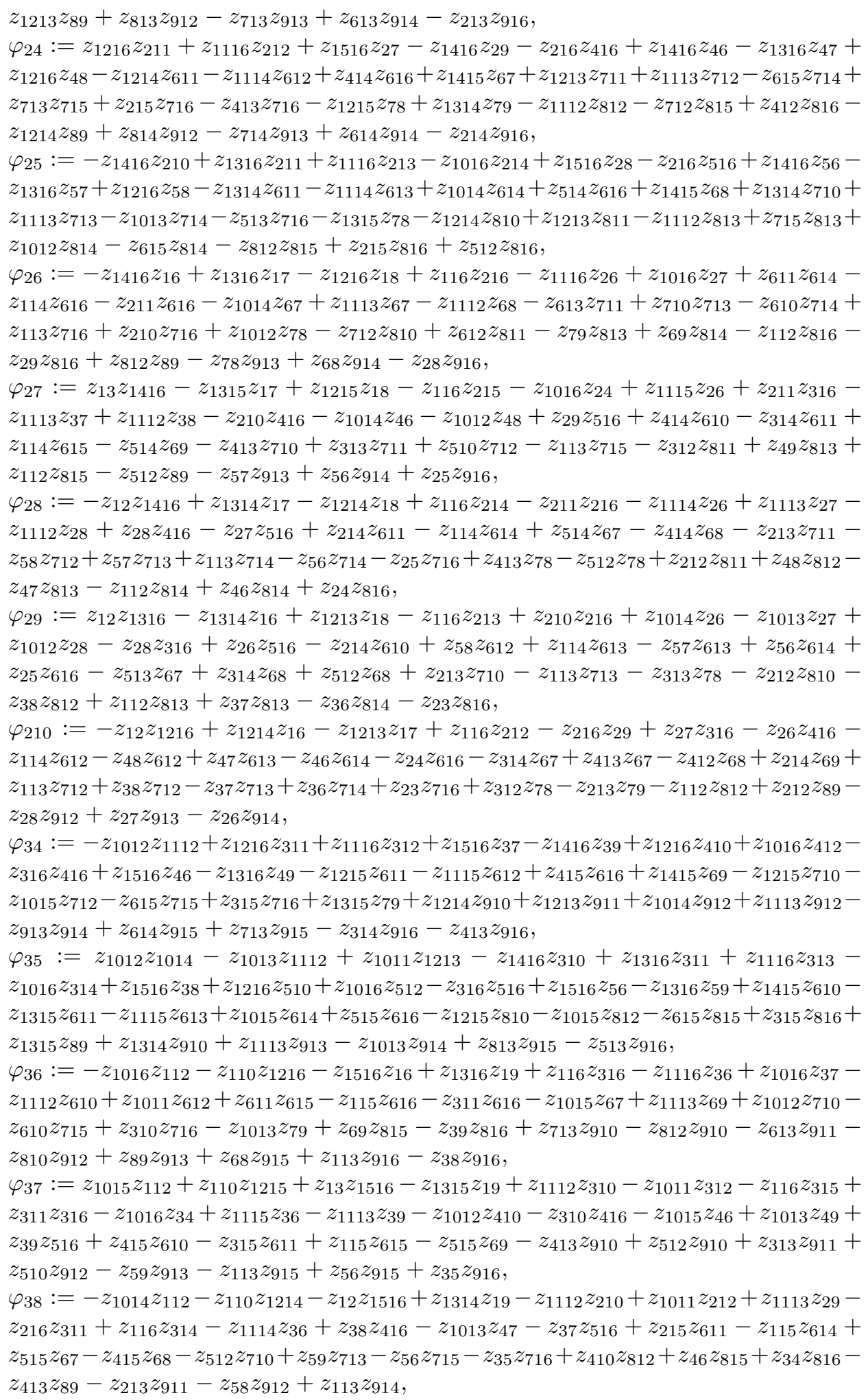




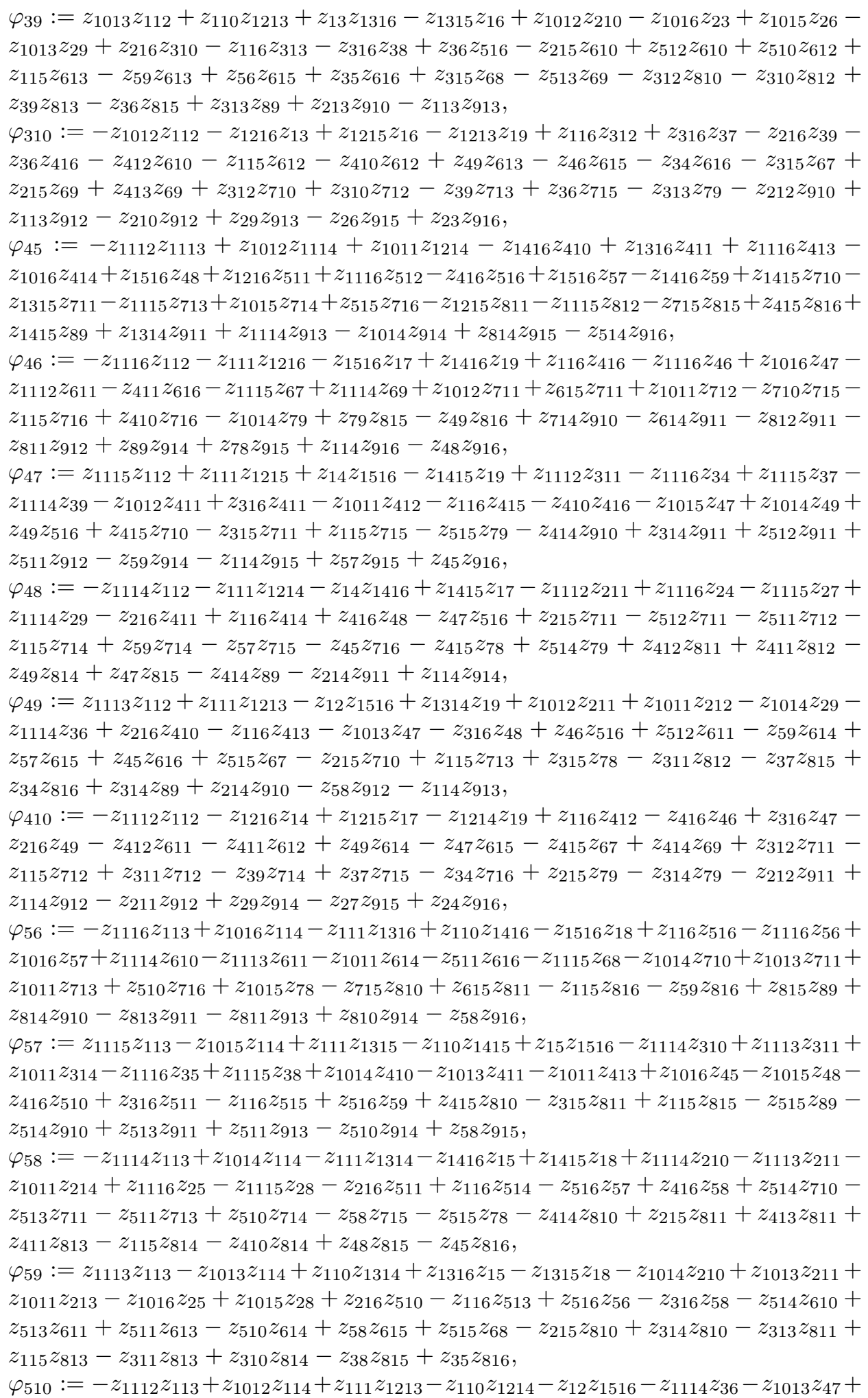




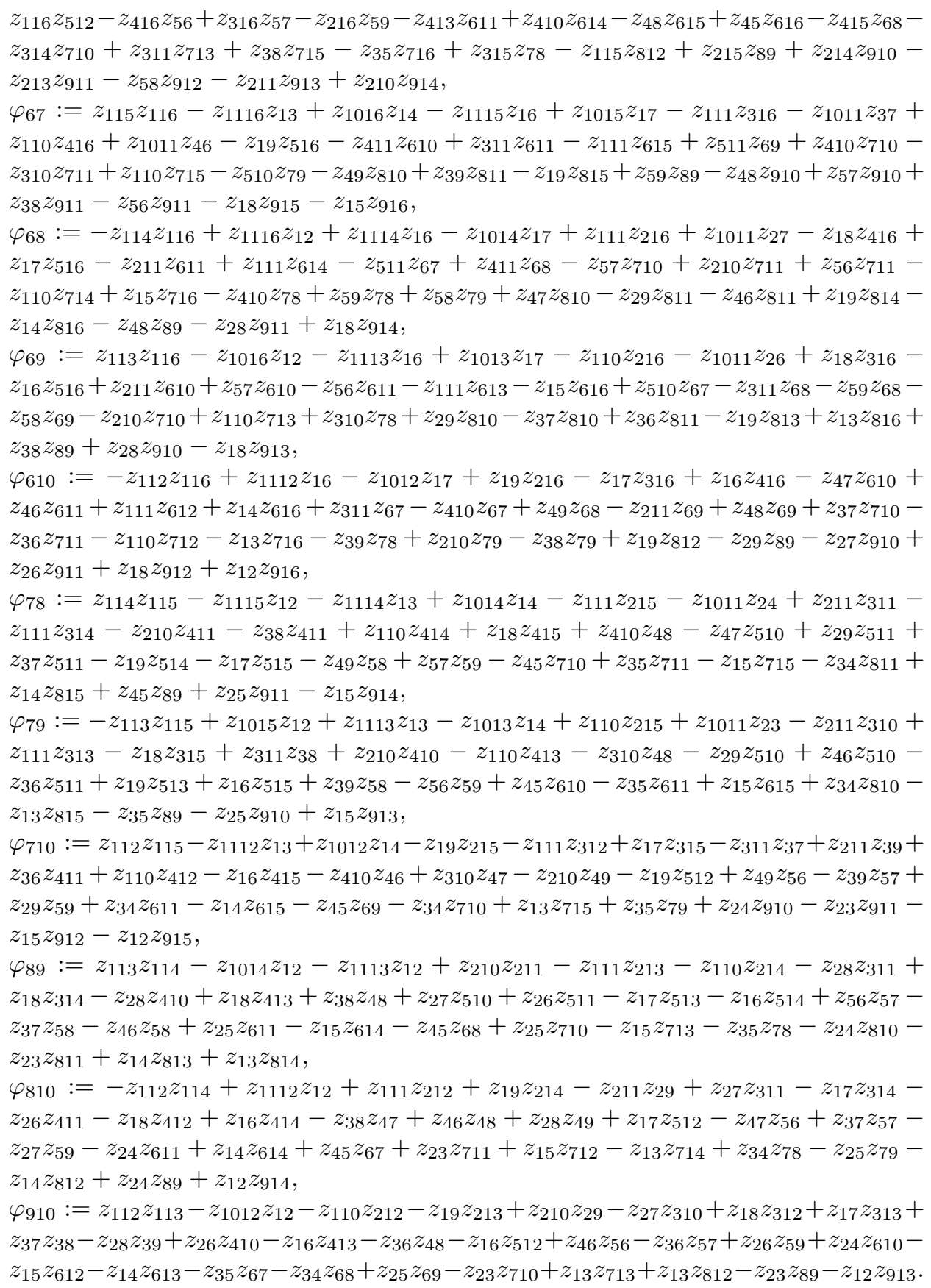

Lemma 3.4. For every $A \in \operatorname{Spin}_{10}, B \in G L_{14}, \tilde{Z} \in \operatorname{Alt}_{16}$, we have $\tilde{Z} \mapsto(\operatorname{det} B) \tilde{Z}$, $\tilde{Z} \mapsto{ }^{t} \Lambda^{\prime}(A)^{-1} \tilde{Z} \Lambda^{\prime}(A)^{-1}$ and hence

$$
\begin{gathered}
\Phi((\operatorname{det} B) \tilde{Z}) \mapsto(\operatorname{det} B)^{2} \Phi(\tilde{Z}), \\
\Phi\left({ }^{t} \Lambda^{\prime}(A)^{-1} \tilde{Z} \Lambda^{\prime}(A)^{-1}\right)={ }^{t} \chi(A)^{-1} \Phi(\tilde{Z}) \chi(A)^{-1},
\end{gathered}
$$


where $\chi$ is the vector representation of $\operatorname{Spin}_{10}$ in (3.9). Hence we have, by the action of $(\alpha, A, B) \in G L_{1} \times \operatorname{Spin}_{10} \times G L_{14}$,

$$
\Phi(\tilde{Z}) \mapsto(\operatorname{det} B)^{t} \chi(A)^{-1} \Phi(\tilde{Z}) \chi(A)^{-1}
$$

Proof. We may prove the equivariance (3.10) for $A^{\prime} s$ of one of the following three forms:
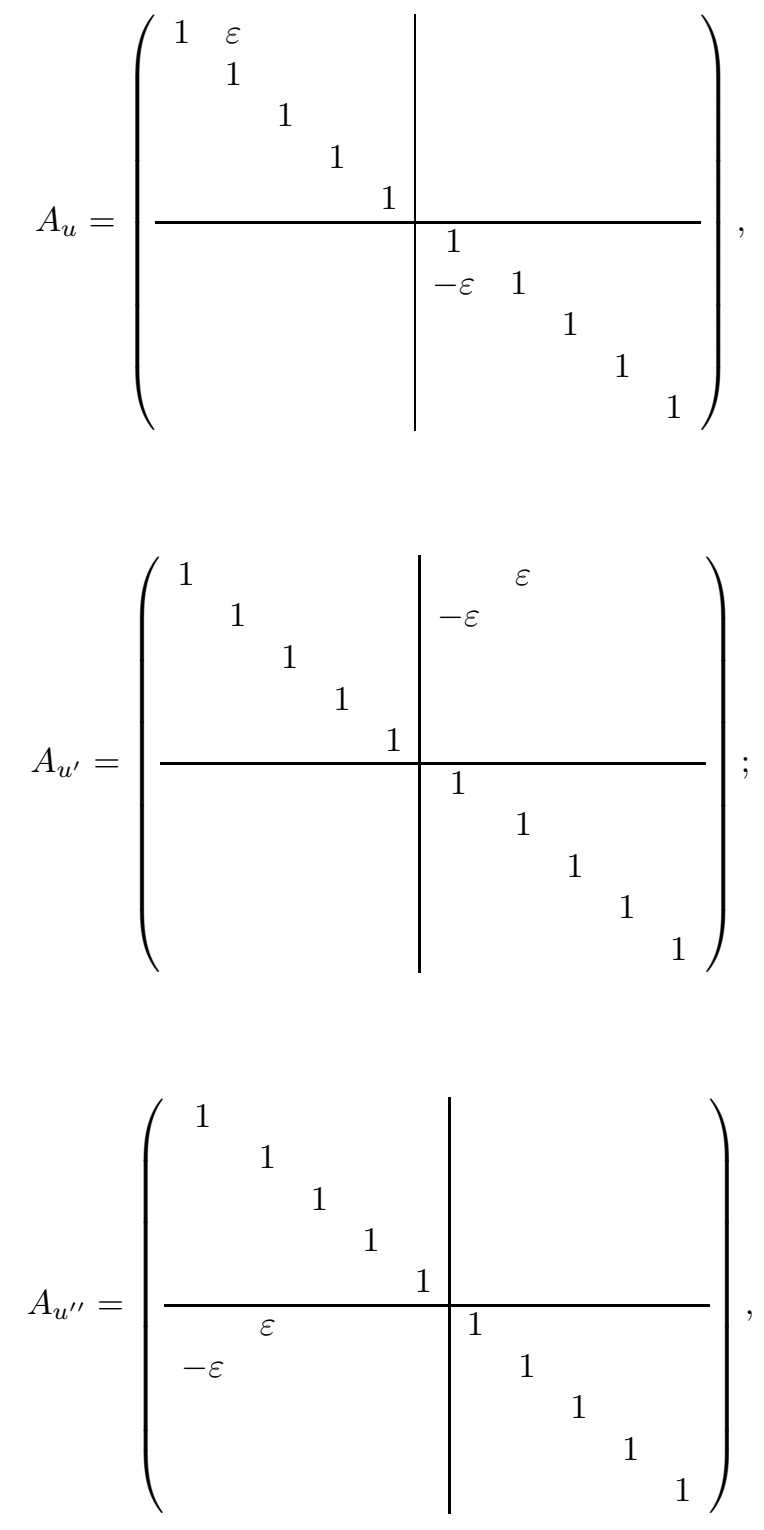

$$
A_{d}=\operatorname{diag}\left(a_{1}, a_{2}, a_{3}, a_{4}, a_{5}, a_{1}^{-1}, a_{2}^{-1}, a_{3}^{-1}, a_{4}^{-1}, a_{5}^{-1}\right)
$$


and permutation type matrices such as

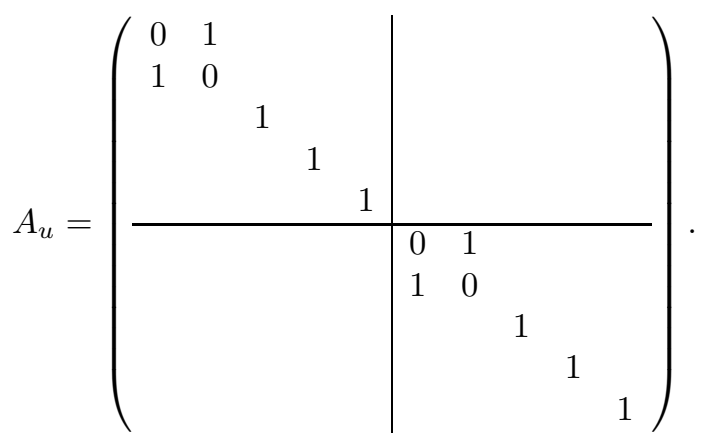

Checking (3.10), for diagonal or permutation type matrices is easy. Note that, for $A_{d}, \quad z_{12} \mapsto a_{3} a_{4} a_{5} z_{12}, z_{13} \mapsto a_{2} a_{4} a_{5} z_{13}, \ldots, z_{23} \mapsto a_{1}^{-1} a_{4} a_{5} z_{23}, z_{24} \mapsto$ $a_{1}^{-1} a_{3} a_{5} z_{24}, \ldots, z_{1516} \mapsto a_{3}^{-1} a_{4}^{-1} a_{5}^{-1} z_{1516}$. Hence $\varphi_{i j} \mapsto a_{i} a_{j} \varphi_{i j}$ for $1 \leq i \leq j \leq 5$, $\varphi_{l k} \mapsto a_{l}^{-1} a_{k}^{-1} \varphi_{l k}$ for $6 \leq l \leq k \leq 10, \varphi_{l j} \mapsto a_{l}^{-1} a_{j} \varphi_{l j}$ for $6 \leq l \leq 10,1 \leq j \leq 5$. Then we have $\Phi\left({ }^{t} \Lambda^{\prime}\left(A_{d}\right)^{-1} \tilde{Z} \Lambda^{\prime}\left(A_{d}\right)^{-1}\right)={ }^{t} \chi\left(A_{d}\right)^{-1} \Phi(\tilde{Z}) \chi\left(A_{d}\right)^{-1}$. For $A_{u}$, we consider the action of $A_{u}$. Since $\operatorname{det} A_{u}=1, \quad \Phi(\tilde{Z}) \mapsto \Phi\left({ }^{t} \Lambda^{\prime}\left(A_{u}\right) \tilde{Z} \Lambda^{\prime}\left(A_{u}\right)^{-1}\right)$. Then we have $\varphi_{11} \mapsto \varphi_{11}+2 \varepsilon \varphi_{12}+\varepsilon^{2} \varphi_{22}, \quad \varphi_{1 j} \mapsto \varphi_{1 j}+\varepsilon \varphi_{2 j} \quad(1 \leq j \leq 5), \quad \varphi_{77} \mapsto$ $\varphi_{77}-2 \varepsilon \varphi_{67}+\varepsilon^{2} \varphi_{66}, \quad \varphi_{67} \mapsto \varphi_{67}-\varepsilon \varphi_{66}, \quad \varphi_{7 k} \mapsto \varphi_{7 k}-\varepsilon \varphi_{6 k} \quad(8 \leq k \leq 10), \quad \varphi_{17} \mapsto$ $\varphi_{17}+\varepsilon\left(\varphi_{27}-\varphi_{16}\right)+\varepsilon^{2} \varphi_{26}, \quad \varphi_{1 j} \mapsto \varphi_{1 j}+\varepsilon \varphi_{2 j} \quad(j \in\{6,8,9,10\}), \quad \varphi_{k 7} \mapsto$ $\varphi_{k 7}-\varepsilon \varphi_{k 6} \quad(k \in\{2,3,4,5\})$ and other $\varphi_{i j}$ 's are invariants. Thus, we have $\Phi\left({ }^{t} \Lambda^{\prime}\left(A_{u}\right)^{-1} \tilde{Z} \Lambda^{\prime}\left(A_{u}\right)^{-1}\right)={ }^{t} \chi\left(A_{u}\right)^{-1} \Phi(\tilde{Z})^{t} \chi\left(A_{u}\right)^{-1}$.

In the same way, we have $\Phi\left({ }^{t} \Lambda^{\prime}\left(A_{u^{\prime}}\right)^{-1} \tilde{Z} \Lambda^{\prime}\left(A_{u^{\prime}}\right)^{-1}\right)={ }^{t} \chi\left(A_{u^{\prime}}\right)^{-1} \Phi(\tilde{Z}) \chi\left(A_{u^{\prime}}\right)^{-1}$ and $\Phi\left({ }^{t} \Lambda^{\prime}\left(A_{u^{\prime \prime}}\right)^{-1} \tilde{Z} \Lambda^{\prime}\left(A_{u^{\prime \prime}}\right)^{-1}\right)={ }^{t} \chi\left(A_{u^{\prime \prime}}\right)^{-1} \Phi(\tilde{Z}) \chi\left(A_{u^{\prime \prime}}\right)^{-1}$.

Remark $\mathrm{C}$. We can construct the above polynomials $\varphi_{i j}(1 \leq i \leq j \leq 10)$ in the same program as in Remark $\mathrm{A}$ in $\S \S 3.1$. We have

$$
K \Phi(\tilde{Z}) \mapsto(\operatorname{det} B)^{2} K^{t} \chi(A)^{-1} \Phi(\tilde{Z}) \chi(A)^{-1}=(\operatorname{det} B)^{2} \chi(A) K \Phi(\tilde{Z}) \chi(A)^{-1}
$$

with $K=\left(\begin{array}{c|c}0 & I_{5} \\ \hline I_{5} & 0\end{array}\right)$ and hence $F_{1}(x)=\operatorname{tr} K \Phi(\tilde{Z})$ is a relative invariant of degree 28 corresponding to the character $(\operatorname{det} B)^{2}$. Moreover, by $(3.7)$ and Lemma 3.4, we put $F_{2}(x)=\langle\eta(X \cdot Y)|\Phi(\tilde{Z})| \eta(X \cdot Y)\rangle:={ }^{t} \eta(X \cdot Y) \Phi(\tilde{Z}) \eta(X \cdot Y)$ and hence $F_{2}(x)$ is a relative invariant of degree 36 corresponding to the character $\alpha^{4}(\operatorname{det} B)^{2}$.

For a generic point

$$
\begin{array}{r}
x_{0}=\left(e_{1} e_{5}+e_{2} e_{3} e_{4} e_{5}, e_{2} e_{5}, e_{3} e_{5}, e_{4} e_{5},-e_{1} e_{3} e_{4} e_{5}, e_{1} e_{2} e_{4} e_{5},-e_{1} e_{2} e_{3} e_{5},\right. \\
\left.-1+e_{1} e_{2} e_{3} e_{4}, e_{1} e_{2}, e_{1} e_{3}, e_{1} e_{4},-e_{3} e_{4}, e_{2} e_{4},-e_{2} e_{3}, e_{4}^{\prime}+e_{14}^{\prime}\right),
\end{array}
$$

we have

$$
\begin{gathered}
X_{0} \cdot Y_{0}={ }^{t}(0,0,0,0,0,-1,0,0,0,0,1,0,0,0,0,0), \\
\eta\left(X_{0} \cdot Y_{0}\right)={ }^{t}(0,0,0,0,0,1,0,0,0,0), \\
\tilde{Z}_{0}=\left(e_{1} \wedge e_{5}-e_{1} \wedge e_{16}\right) \in \mathrm{Alt}_{16},
\end{gathered}
$$




$$
\Phi\left(\tilde{Z}_{0}\right)=\Phi\left(\tilde{Z}_{0}\right)=\left(\begin{array}{ccccc|ccccc}
-1 & 0 & 0 & 0 & 0 & -1 & 0 & 0 & 0 & 0 \\
0 & 0 & 0 & 0 & 0 & 0 & 0 & 0 & 0 & 0 \\
0 & 0 & 0 & 0 & 0 & 0 & 0 & 0 & 0 & 0 \\
0 & 0 & 0 & 0 & 0 & 0 & 0 & 0 & 0 & 0 \\
0 & 0 & 0 & 0 & 0 & 0 & 0 & 0 & 0 & -1 \\
\hline-1 & 0 & 0 & 0 & 0 & -1 & 0 & 0 & 0 & 0 \\
0 & 0 & 0 & 0 & 0 & 0 & 0 & 0 & 0 & 0 \\
0 & 0 & 0 & 0 & 0 & 0 & 0 & 0 & 0 & 0 \\
0 & 0 & 0 & 0 & 0 & 0 & 0 & 0 & 0 & 0 \\
0 & 0 & 0 & 0 & -1 & 0 & 0 & 0 & 0 & 0
\end{array}\right) \in \text { Sym }_{10} .
$$

Then we have $F_{2}\left(X_{1}^{(0)}, \cdots, X_{14}^{(0)}, Y_{0}\right)=\left\langle\eta\left(X_{0} \cdot Y_{0}\right)\left|\Phi\left(\tilde{Z}_{0}\right)\right| \eta\left(X_{0} \cdot Y_{0}\right)\right\rangle=-1 \neq$ 0 and $F_{1}\left(X_{1}^{(0)}, \cdots, X_{14}^{(0)}, Y_{0}\right)=\operatorname{tr} K \Phi\left(\tilde{Z}_{0}\right)=-4 \neq 0$ and hence they are not identically zero.

In [6] or [8, the irreducible relative invariant $f(x)$ of degree 4 of a prehomogeneous vector space $\left(\operatorname{Spin}_{10} \times G L_{2}, \Lambda^{\prime} \otimes \Lambda_{1}, V(16) \oplus V(16)\right)$ is constructed. Through the theory of castling transformation, above polynomial $F_{1}(x)$ is corresponding to the irreducible relative invariant of a prehomogeneous vector space $\left(\operatorname{Spin}_{10} \times G L_{2}, \Lambda^{\prime} \otimes \Lambda_{1}, V(16) \oplus V(16)\right)$ studied in [6]. By Lemma 1.2, we can know the irreducibility of $F_{1}(x)$. Note that we can also check the degree of $F_{1}(x)$ by compairing the degree of $f(x)$ by Lemma 1.2 .

If the polynomial $F_{2}(x)$ is not irreducible, then $F_{2}(x)=F_{1}(x) G(x)$ for some relative invariant $G(x)$ of degree 8 corresponding to the character $\alpha^{4}$, or $F_{2}(x)=$ $H(x)^{2}$ for some relative invariant $H(x)$ of degree 18 corresponding to the character $\alpha^{2}(\operatorname{det} B)$. For $x_{1}={ }^{t}\left(t X_{1}^{(0)}, X_{2}^{(0)}, \ldots, X_{14}^{(0)}, Y_{1}\right) \in V(16)^{\oplus 14} \oplus V(14)^{*}$ with $Y_{1}={ }^{t}\left(y_{1}, 0,0, y_{4}, 0,0,0, y_{8}, 0,0, y_{11}, 0,0, y_{14}\right)$, we have $F_{1}\left(x_{1}\right)=-4 t^{2}$ and $F_{2}\left(x_{1}\right)=$ $2 t^{2} y_{1}^{2} y_{11}^{2} y_{14}-y_{14}^{2} y_{4}^{2}-2 t^{2} y_{1}^{2} y_{8}^{2}$. Then we have $F_{1}\left(x_{1}\right) \nmid F_{2}\left(x_{1}\right)$ and hence $F_{1}(x) \nmid F_{2}(x)$. Moreover, $F_{2}\left(x_{1}\right)$ cannot be of the form $F_{2}\left(x_{1}\right)=H\left(x_{1}\right)^{2}$, and hence $F_{2}(x)$ cannot be of the form $F_{2}(x)=H(x)^{2}$. Thus we have obtained the irreducibility of $F_{2}(x)$.

Theorem 3.5. The prehomogeneous vector space $\left(G L_{1} \times \operatorname{Spin}_{10} \times G L_{14}, \Lambda^{\prime} \otimes \Lambda_{1}+\right.$ $\left.1 \otimes \Lambda_{1}^{*}, V(16)^{\oplus 14} \oplus V(14)^{*}\right)$ has 2 basic relative invariants:

(1) $F_{1}(x)=\operatorname{tr} K \Phi(\tilde{Z}) \longleftrightarrow(\operatorname{det} B)^{2}, \operatorname{deg} \cdot F_{1}=28$,

(2) $F_{2}(x)=\langle\eta(X \cdot Y)|\Phi(\tilde{Z})| \eta(X \cdot Y)\rangle \longleftrightarrow \alpha^{4}(\operatorname{det} B)^{2}, \operatorname{deg} \cdot F_{2}=36$.

\section{ACKNOWLEDGMENT}

The authors would like to express their hearty thanks to Professor H. Ochiai for his invaluable comments for this paper.

\section{REFERENCES}

[1] M. Sato and T. Kimura, A classification of irreducible prehomogeneous vector spaces and their relative invariants, Nagoya Math. J., 65, (1977), 1-155. MR 55:3341

[2] T. Kimura, A classification of prehomogeneous vector spaces of simple algebraic groups with scalar multiplications, J. Algebra, 83, No. 1, (1983), 72-100. MR 85d:32059

[3] T. Kimura, Remark on some combinatrial construction of relative invariants, Tsukuba J. Math. 5, (1981), 101-115. MR 84g:20078

[4] J. Igusa, A classification on spinors up to dimension twelve, Amer. J. Math. 92, (1970), 997-1028. MR 43:3291

[5] J. Igusa, On arithmetic of a singular invariant, Amer. J. Math. 110, (1988), 197-233. MR 86g:11116 
[6] H. Kawahara, Prehomogeneous vector spaces related with the spin group, Master Thesis, University of Tokyo, (1974) (Japanese).

[7] A. Gyoja, Construction of invariants, Tsukuba J. Math. 14, (1990), No. 2, 437-457. MR 91k:22035

[8] H. Ochiai, Quotients of some prehomogeneous vector spaces, J. Algebra, 192, (1997), No. 1, 61-73. MR 98g:14059

[9] T. Kimura, S. Kasai, M. Inuzuka and O. Yasukura, A classification of 2-simple prehomogeneous vector spaces of type I, J. Algebra, 114, No. 2, (1988), 369-400. MR 89f:32062

[10] T. Kogiso, G. Miyabe, M. Kobayashi and T. Kimura, Explicit construction of relative invariants for regular 2-simple prehomogeneous vector spaces of type I, preprint

[11] T. Kogiso, G. Miyabe, M. Kobayashi and T. Kimura, Nonregular 2-simple prehomogeneous vector spaces of type I and their relative invariants, J. Algebra, 251, 27-69 (2002).

[12] Mathematica, Wolfram Research Inc.

The Department of Mathematics, Josai University, Saitama, 305-0295, Japan

E-mail address: kogiso@math.josai.ac.jp

The Institute of Mathematics, University of Tsukuba, Ibaraki, 305-8571, Japan

E-mail address: subaru@first.tsukuba.ac.jp

The Institute of Mathematics, University of Tsukuba, Ibaraki, 305-8571, Japan

E-mail address: miyuki@math.tsukuba.ac.jp

The Institute of Mathematics, University of Tsukuba, Ibaraki, 305-8571, Japan

E-mail address: kimurata@math.tsukuba.ac.jp 